\title{
Surface links which are coverings over the standard torus
}

\author{
INASA NAKAMURA
}

\begin{abstract}
We introduce a new construction of a surface link in 4-space. We construct a surface link as a branched covering over the standard torus, which we call a torus-covering link. We show that a certain torus-covering $T^{2}-$ link is equivalent to the split union of spun $T^{2}$-links and turned spun $T^{2}$-links. We show that a certain torus-covering $T^{2}-$ link has a nonclassical link group. We give a certain class of ribbon torus-covering $T^{2}$-links. We present the quandle cocycle invariant of a certain torus-covering $T^{2}$ link obtained from a classical braid, by using the quandle cocycle invariants of the closure of the braid.
\end{abstract}

57Q45; 57Q35

\section{Introduction}

A surface link is the image of a smooth embedding of a closed surface into the Euclidean 4 -space $\mathbb{R}^{4}$. It is known (see Kamada $[22 ; 24]$ ) that any oriented surface link can be presented by the closure of a surface braid. Here, the closure of a surface braid is a surface link of the following form. Let $S^{2}$ be a standard 2-sphere in $\mathbb{R}^{4}$, ie the boundary of a standard 3-ball in $\mathbb{R}^{3} \times\{0\}$. The closure of a surface braid is a surface link embedded in a tubular neighborhood $N\left(S^{2}\right)$ of $S^{2}$ in such a way that the projection of it to $S^{2}$ is a branched covering over $S^{2}$. We identify $N\left(S^{2}\right)$ with $I \times I \times S^{2}$, where $I$ is an interval. For a surface link $S$ of such a form, we consider the singular set of the image of $S$ by the projection to $I \times S^{2}$, and the image of this singular set by the projection to $S^{2}$ forms a graph on $S^{2}$. An $m$-chart on $S^{2}$ is such a graph with certain additional data. We can present the original surface link by its $m$-chart on $S^{2}$ (see Kamada [23; 24]).

In this paper we introduce a "torus-covering link" as a new construction of a surface link, by considering a standard torus instead of a standard 2-sphere. Let $T$ be a standard torus in $\mathbb{R}^{4}$, ie the boundary of a standard solid torus in $\mathbb{R}^{3} \times\{0\}$. A torus-covering link is a surface link embedded in a tubular neighborhood $N(T)$ of $T$ in such a way that the projection of it to $T$ is a branched covering over $T$. For a surface link of such a form, we can define its $m$-chart on $T$ in the same way as above. A torus-covering 
link can be presented by an $m$-chart on $T$. The aim of this paper is to study various aspects of torus-covering links.

We introduce an equivalence relation, called the $t$-equivalence, among $m$-charts on $T$, and show that two torus-covering links are equivalent if their $m$-charts on $T$ are $t$-equivalent (Theorem 2.5). A $T^{2}$-link is a surface link whose components are homeomorphic to tori. We show that a torus-covering $T^{2}$-link is determined from two commutative classical $m$-braids (Lemma 2.8), which we call basis $m$-braids, and we denote by $\mathcal{S}_{m}(a, b)$ the torus-covering $T^{2}$-link with basis $m$-braids $a$ and $b$. A vertex of degree one (respectively six) of an $m$-chart is called a black vertex (respectively a white vertex). A torus-covering $T^{2}$-link is presented by an $m$-chart on $T$ without black vertices (Lemma 2.7). We show that an $m$-chart on $T$ with neither black nor white vertices presents the split union of spun $T^{2}$-links and turned spun $T^{2}-$ links (Theorem 2.16).

The link group of a surface link or a classical link is the fundamental group of the link exterior. First we calculate the link group of $\mathcal{S}_{m}(a, b)$ (Proposition 3.1). It is known (see Livingston [26] and Boyle [8]) that a spun $T^{2}-$ link or a turned spun $T^{2}$-link has a classical link group; thus the split union of spun $T^{2}$-links and turned spun $T^{2}$-links also has a classical link group. We will show that a certain 2-component torus-covering $T^{2}$-link has a nonclassical link group (Theorem 3.2). We show its knot version as well: a certain torus-covering $T^{2}-$ knot has a nonclassical knot group (Theorem 3.7). As a corollary, we can see that the torus-covering $T^{2}$-link of Theorems 3.2 or 3.7 is not equivalent to the split union of spun $T^{2}$-links and turned spun $T^{2}$-links (Theorem 3.12).

An oriented surface link is called ribbon if it is the boundary of an immersed 3-manifold with "ribbon singularities" (see Yanagawa [32]). We give a certain class of ribbon toruscovering $T^{2}$-links (Theorem 4.1). As a corollary, we can see that the torus-covering $T^{2}$-link of Theorem 3.12 is ribbon (Corollary 4.2).

It is known (see Asami and Satoh [2]) that the quandle cocycle invariant of a twist spun 2-knot of a classical knot $K$ can be presented by using the quandle cocycle invariants of a 1-tangle whose closure is $K$. From a similar viewpoint, we expect that an invariant of $\mathcal{S}_{m}\left(b, \Delta^{2 n}\right)$ can be presented by using invariants of an $m$-braid $b$, where $\Delta$ is a half-twist of a bundle of $m$ parallel strands. In Theorem 5.2 we present the quandle cocycle invariant of $\mathcal{S}_{m}\left(b, \Delta^{2 n}\right)$, by using the quandle cocycle invariants of the closure of $b$. In Theorem 5.5, we calculate some concrete examples of Theorem 5.2. They give torus-covering $T^{2}$-knots whose triple point numbers are positive (Corollary 5.6).

The paper is organized as follows. In Section 2, we define a torus-covering link (Definition 2.3) and show Theorem 2.5. Further we study torus-covering $T^{2}-$ links and 
show Theorem 2.16. In Section 3, we study link groups of torus-covering $T^{2}$-links. We show Theorem 3.2 and Theorem 3.7. Further we show Theorem 3.12. In Section 4, we show Theorem 4.1. In Section 5, we calculate the quandle cocycle invariants and show Theorem 5.2. Further we show Theorem 5.5, by using Mochizuki's 3-cocycle.

\section{Torus-covering links}

A braided surface over a 2-disk was defined by Rudolph [30] and Kamada [24]. A surface braid is a braided surface with some boundary condition, and a notion of an $m$-chart on a 2-disk was introduced by Kamada [20;24] to present a simple surface braid. Equivalent simple surface braids have distinct chart presentations. The notion of C-move equivalence between two $m$-charts on a 2-disk was introduced by Kamada [20; $23 ; 24]$ to give the equivalence class of an $m$-chart which represents the equivalence class of a simple surface braid. In this section, we modify the definitions to define a braided surface $S$ over a closed surface $\Sigma$, an $m$-chart on $\Sigma$ which presents $S$, and the notion of $\mathrm{C}$-move equivalence between two $m$-charts on $\Sigma$. Using these terms, we define a torus-covering link, which is presented by an $m$-chart on the standard torus. We define $t$-equivalence between two $m$-charts, and show that the torus-covering links are equivalent if $m$-charts of them are $t$-equivalent (Theorem 2.5). Further we study torus-covering $T^{2}$-links. A torus-covering $T^{2}$-link is presented by an $m$-chart on $T$ without black vertices (Lemma 2.7). We show Theorem 2.16: an $m$-chart on $T$ with neither black nor white vertices presents the split union of spun $T^{2}$-links and turned spun $T^{2}-$ links.

We work in the smooth category, and we assume that embeddings are locally flat. Let $D^{2}=I \times I$, where $I=[0,1]$. A surface link is the image of a smooth embedding of a closed surface into $\mathbb{R}^{4}$. Two surface links are said to be equivalent if one is taken to the other by an orientation-preserving self-diffeomorphism of $\mathbb{R}^{4}$.

Definition 2.1 A closed surface $S$ embedded in $D^{2} \times \Sigma$ is called a braided surface over $\Sigma$ of degree $m$ if $\left.p_{\Sigma}\right|_{S}: S \rightarrow \Sigma$ is a branched covering map of degree $m$, where $p_{\Sigma}: D^{2} \times \Sigma \rightarrow \Sigma$ is the projection to the second factor. A braided surface $S$ is called simple if $\#\left(S \cap p_{\Sigma}^{-1}(x)\right)=m-1$ or $m$ for each $x \in \Sigma$. Take a base point $x_{0}$ of $\Sigma$. Two braided surfaces over $\Sigma$ of degree $m$ are equivalent if there is a fiber-preserving ambient isotopy of $D^{2} \times \Sigma$ rel $p_{\Sigma}^{-1}\left(x_{0}\right)$ which carries one to the other.

When a simple braided surface $S$ is given, we obtain a graph on $\Sigma$, as follows. Consider the singular set $\operatorname{Sing}\left(p_{1}(S)\right)$ of the image of $S$ by the projection $p_{1}$ to $I \times \Sigma$. Perturbing $S$ if necessary, we can assume that $\operatorname{Sing}\left(p_{1}(S)\right)$ consists of double 
point curves, triple points, and branch points. Moreover we can assume that the singular set of the image of $\operatorname{Sing}\left(p_{1}(S)\right)$ by the projection to $\Sigma$ consists of a finite number of double points such that the preimages belong to double point curves of $\operatorname{Sing}\left(p_{1}(S)\right)$. Thus the image of $\operatorname{Sing}\left(p_{1}(S)\right)$ by the projection to $\Sigma$ forms a finite graph $\Gamma$ on $\Sigma$ such that the degree of its vertex is either 1,4 or 6 . An edge of $\Gamma$ corresponds to a double point curve, and a vertex of degree 1 (respectively 6) corresponds to a branch point (respectively a triple point).

For such a graph $\Gamma$ obtained from a simple braided surface $S$, we give orientations and labels to the edges of $\Gamma$, as follows. Let us consider a path $l$ in $\Sigma$ such that $l \cap \Gamma$ is a point $P$ of an edge $e$ of $\Gamma$. Then $S \cap p_{\Sigma}^{-1}(l)$ is a classical $m$-braid with one crossing in $p_{\Sigma}^{-1}(l)$ such that $P$ corresponds to the crossing of the $m$-braid. Let $\sigma_{1}, \sigma_{2}, \ldots, \sigma_{m-1}$ be the standard generators of the $m$-braid group $B_{m}$. Let $\sigma_{i}^{\epsilon}$ $(i \in\{1,2, \ldots, m-1\}, \epsilon \in\{+1,-1\})$ be the presentation of $S \cap p_{\Sigma}^{-1}(l)$. Then label the edge $e$ by $i$, and moreover give $e$ an orientation such that the normal vector of $l$ corresponds (respectively does not correspond) to the orientation of $e$ if $\epsilon=+1$ (respectively -1 ). We call such an oriented and labeled graph an $m$-chart of $S$.

In general, we define an $m$-chart on $\Sigma$ as follows.

Definition 2.2 Let $m$ be a positive integer, and let $\Gamma$ be a finite graph on $\Sigma$. Then $\Gamma$ is called an $m$-chart on $\Sigma$ if it satisfies the following conditions:

(i) Every edge is oriented and labeled by an element of $\{1,2, \ldots, m-1\}$.

(ii) Every vertex has degree 1, 4 or 6 .

(iii) The adjacent edges around each vertex are oriented and labeled as shown in Figure 1, where we depict a vertex of degree 1 by a black vertex, and a vertex of degree 6 by a white vertex.
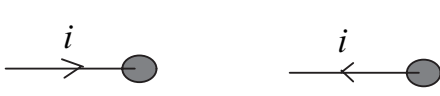

black vertex

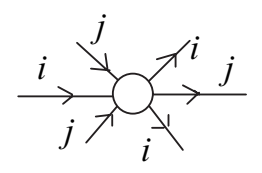

$$
|i-j|=1
$$

white vertex

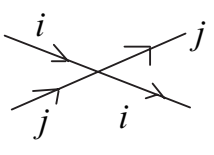

$|i-j|>1$

Figure 1: Vertices in an $m$-chart

When an $m$-chart $\Gamma$ on $\Sigma$ is given, we can reconstruct a simple braided surface $S$ over $\Sigma$ as follows. Let $N(\Gamma)$ be a neighborhood of $\Gamma$ in $\Sigma$. Let us consider a trivial 
braided surface $S=Q_{m} \times(\Sigma-N(\Gamma))$ over $\Sigma-N(\Gamma)$, where $Q_{m}$ is a set of $m$ interior points of $D^{2}$. We extend $S$ over a neighborhood of each edge as follows. Identify a neighborhood of an edge $e$ with $I \times I$ such that $e$ is identified with $\{1 / 2\} \times I$. Let $i$ be the label attached to $e$, and let $\epsilon=+1$ (respectively -1 ) if the orientation of $e$ corresponds (respectively does not correspond) to the orientation of $\{0\} \times I$. Then let the braided surface $S$ over the neighborhood of $e$ be the braided surface which has a presentation $\sigma_{i}^{\epsilon} \times I$ and the image of the double point curve of $p_{1}(S)$ by the projection to $\Sigma$ is $e$. Since $\Gamma$ is as in Figure 1 around each vertex, $S$ can be extended naturally over a neighborhood of each vertex. See Carter and Saito [14] and Kamada $[21 ; 24]$ for more details. Thus we can construct a simple braided surface $S$ over $\Sigma$ such that the original $m$-chart $\Gamma$ is an $m$-chart of $S$.

Two $m$-charts on $\Sigma$ are $C$-move equivalent if they are related by a finite sequence of ambient isotopies of $\Sigma$ rel $p_{\Sigma}^{-1}\left(x_{0}\right)$ and CI, CII, CIII-moves shown in Figure 2; see [24] for the complete set of CI-moves. It is shown as a minor modification of [20; $23 ; 24]$ that two simple braided surfaces over $\Sigma$ of degree $m$ are equivalent if and only if $m$-charts of them are $\mathrm{C}$-move equivalent.

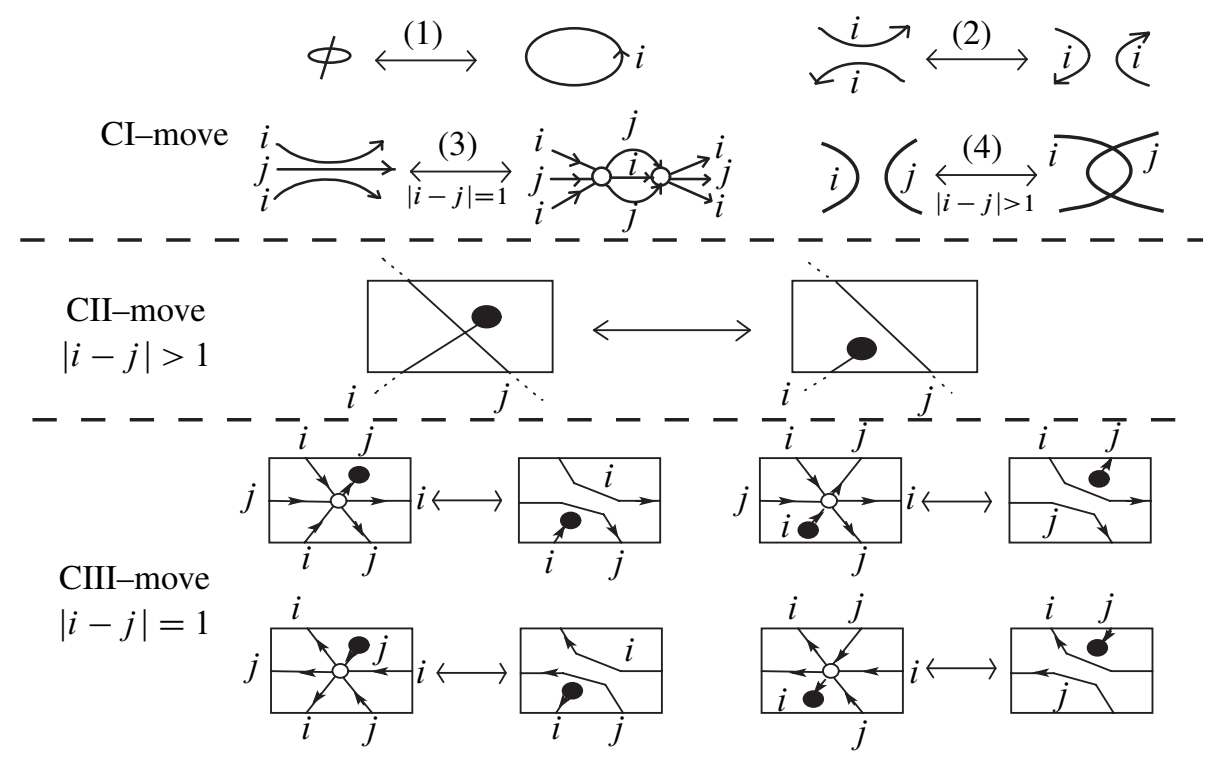

Figure 2: CI, CII, CIII-moves. For CI-moves, we give only several examples.

Now we define torus-covering links. Let $T$ be the standard torus in $\mathbb{R}^{4}$, ie the boundary of the standard solid torus in $\mathbb{R}^{3} \times\{0\}$. Let us fix a point $x_{0}$ of $T$, and take a meridian $\mathbf{m}$ and a longitude $\mathbf{l}$ of $T$ with the base point $x_{0}$. A meridian is an oriented simple closed curve on $T$ which bounds the 2-disk of the solid torus whose boundary 
is $T$. A longitude is an oriented simple closed curve on $T$ which is null-homologous in the complement of the solid torus in the three space $\mathbb{R}^{3} \times\{0\}$. Let $N(T)$ be a tubular neighborhood of $T$ in $\mathbb{R}^{4}$. Since $T$ is the boundary of the standard solid torus in $\mathbb{R}^{3} \times\{0\}$, the normal bundle of $T$ in $\mathbb{R}^{3} \times\{0\}$ is a trivial bundle. Let us identify it with $I \times T$. Then let us identify $N(T)$ with $I \times I \times T$, where the second $I$ is an interval in the fourth axis of $\mathbb{R}^{4}$. From now on, we identify $N(T)$ with $D^{2} \times T$.

Definition 2.3 A torus-covering link is a surface link in $\mathbb{R}^{4}$ presented by a simple braided surface over $T$, where we regard the braided surface as in $N(T) \subset \mathbb{R}^{4}$.

As we mentioned, for two $m$-charts on $T$, their presenting braided surfaces over $T$ are equivalent if the $m$-charts are $\mathrm{C}$-move equivalent. Hence it follows that for two $m$-charts on $T$, their presenting torus-covering links are equivalent if the $m$-charts are $\mathrm{C}$-move equivalent. Since each component of a torus-covering link is a branched cover over a torus $T$, each component of a torus-covering link is of genus at least one. See Propositions 2.11, 2.12 and 2.13 for some examples of torus-covering links. Note that it is known (see Berstein and Edmonds $[3 ; 4]$ ) that any braided surface over $T$ is approximated by a simple braided surface over $T$.

Regarding $S^{4}$ as the one-point compactification of $\mathbb{R}^{4}$, we regard a surface link as in $S^{4}$. Then $N(T)=D^{2} \times T$ is embedded in $S^{4}$. Let $\mathbf{r}=\partial D^{2} \times\{0\} \times\{0\}$ be a curve on $\partial N(T)=\partial D^{2} \times T$. Put $E^{4}=\operatorname{cl}\left(S^{4}-N(T)\right)$. Let $r, m$ and $l$ be the curves on $\partial E^{4}$, which are identified with $\mathbf{r}, \mathbf{m}$ and $\mathbf{l}$ under the natural identification map $i: \partial N(T) \rightarrow \partial E^{4}$. The curves $\mathbf{r}, \mathbf{m}$ and $\mathbf{l}$ represent a basis of $H_{1}\left(\partial E^{4} ; \mathbb{Z}\right)$. Let $f: \partial E^{4} \rightarrow E^{4}$ be a diffeomorphism such that $f_{*}(\mathbf{r} \mathbf{~ m ~ l ~})=(\mathbf{r} \mathbf{m ~ l}) A^{f}$, where $A^{f} \in \mathrm{GL}(3, \mathbb{Z}) \cong \pi_{0} \operatorname{Diffeo}\left(\partial E^{4}\right)$. It is known (see Montesinos [28]) that the map $f$ can be extended to a self-diffeomorphism of $E^{4}$ if and only if $A^{f} \in H$, where:

$$
H=\left\{\left(\begin{array}{ccc} 
\pm 1 & 0 & 0 \\
* & \alpha & \gamma \\
* & \beta & \delta
\end{array}\right) \in \operatorname{GL}(3, \mathbb{Z}) ; \alpha+\beta+\gamma+\delta \equiv 0(\bmod 2)\right\}
$$

Using this fact, we introduce an equivalence relation between two $m$-charts on $T$.

Definition 2.4 We say that two $m$-charts on $T$ are $t$-equivalent if they are related by a finite sequence of ambient isotopies of $T, \mathrm{C}$-moves and a self-diffeomorphism of $T$ given by an element of:

$$
\left\{\left(\begin{array}{ll}
\alpha & \gamma \\
\beta & \delta
\end{array}\right) \in \mathrm{GL}_{+}(2, \mathbb{Z}) ; \alpha+\beta+\gamma+\delta \equiv 0(\bmod 2)\right\}
$$


Theorem 2.5 Two torus-covering links in $S^{4}$ are equivalent if $m$-charts of them are $t$-equivalent.

Proof Since C-move equivalent $m$-charts present equivalent torus-covering links, it suffices to show in the cases for an ambient isotopy of $T$ and a self-diffeomorphism $g$ of $T$ of Definition 2.4.

An ambient isotopy of $T$ induces a fiber-preserving ambient isotopy of $N(T)$ which relates the torus-covering links. This can be extended to the whole space by the Isotopy Extension Theorem (see Hirsch [18]). The terminal map of the resulting isotopy is an orientation-preserving diffeomorphism; thus the torus-covering links are equivalent.

Let $\Gamma$ be an $m$-chart on $T$. Let $S$ and $S^{\prime}$ be the torus-covering links presented by $\Gamma$ and $g(\Gamma)$ respectively. Let $g^{\prime}$ be a self-diffeomorphism of $N(T)$ induced by $g$, ie $g^{\prime}=\operatorname{id}_{D^{2}} \times g: N(T) \rightarrow N(T)$, where we regard $N(T)$ as $D^{2} \times T$. Since $A^{g^{\prime}}$ is in $\mathrm{GL}_{+}(3, \mathbb{Z})$, the map $\left.g^{\prime}\right|_{\partial N(T)}$ can be considered as an orientation-preserving self-diffeomorphism of $\partial E^{4}$. Since $A^{g^{\prime}}$ is an element of $H,\left.g^{\prime}\right|_{\partial N(T)}$ can be extended to $E^{4}$, and hence to $S^{4}=N(T) \cup_{\partial N(T)} E^{4}$. This is an orientation-preserving selfdiffeomorphism of $S^{4}$ which maps $S$ to $S^{\prime}$, and hence $S$ and $S^{\prime}$ are equivalent in $S^{4}$.

In particular, we have the following corollary. Let $\rho$, respectively $\tau$, be a selfdiffeomorphism of $T$ given by:

$$
\left(\begin{array}{cc}
0 & -1 \\
1 & 0
\end{array}\right), \quad \text { respectively } \quad\left(\begin{array}{cc}
1 & -1 \\
0 & 1
\end{array}\right)
$$

Corollary 2.6 Two $m$-charts on $T$ present equivalent torus-covering links if they are related by a finite sequence of ambient isotopies of $T, C$-moves, and moves as in Figure 3.

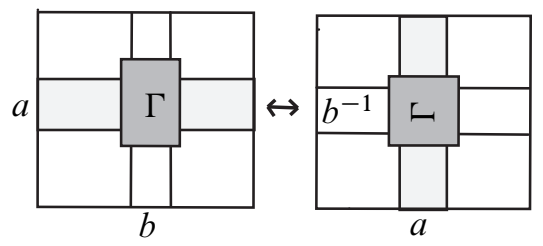

(1)

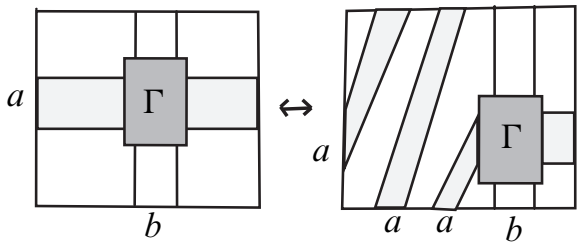

(2)

Figure 3: Equivalent moves of $m$-charts

Proof The moves as in Figure 3 (1) and (2) are related by $\rho$ and $\tau^{2}$ respectively. They give $t$-equivalence between two $m$-charts on $T$. Thus the conclusion follows from Theorem 2.5. 
Remark Teragaito [31] proved the same fact of the above corollary for the symmetryspun version. The case of $\tau^{2}$ for turned spun $T^{2}$-links was shown by Boyle [8], using the result of Gluck [16].

A torus-covering $T^{2}$-link is a torus-covering link whose components are homeomorphic to tori.

Lemma 2.7 A torus-covering $T^{2}$-link $S$ is presented by an $m$-chart without black vertices. Thus $S$ is an unbranched cover over $T$.

Proof Let $\Gamma$ be an $m$-chart on $T$ which presents $S$, and let $b(\Gamma)$ be the number of black vertices in $\Gamma$. Then $S$ is a branched cover over $T$ with $b(\Gamma)$ branch points, and the Euler characteristic of $S$ is equal to $-b(\Gamma)$. Further, since the Euler characteristic of tori is equal to zero, we have $b(\Gamma)=0$.

Let us consider a torus-covering $T^{2}$-link $S$. The intersections $S \cap p_{T}^{-1}(\mathbf{m})$ and $S \cap p_{T}^{-1}(\mathbf{l})$ are closures of classical braids. Cutting open the solid tori at the 2-disk $p_{T}^{-1}\left(x_{0}\right)$, we obtain a pair of classical braids. We call them basis braids.

Lemma 2.8 (1) The basis braids of a torus-covering $T^{2}$-link are commutative.

(2) For any commutative $m$-braids $a$ and $b$, there exists a unique torus-covering $T^{2}$-link with basis braids $a$ and $b$.

For commutative $m$-braids $a$ and $b$, we denote by $\mathcal{S}_{m}(a, b)$ the torus-covering $T^{2}$ link with basis $m$-braids $a$ and $b$.

Proof (1) Let $X_{m}$ be the configuration space of unordered $m$ distinct points of a 2-disk $D^{2}$, ie the set of $m$-element subsets of $D^{2}$ such that each $m$-element subset consists of $m$ distinct points. It is known (see Artin [1] and also Birman [5]) that $\pi_{1}\left(X_{m}\right)=B_{m}$. Since a torus-covering $T^{2}$-link is presented by an unbranched covering of degree $m$ over $T$ by Lemma 2.7, it is presented by a map $f: T \rightarrow X_{m}$. The induced map $f_{*}$ gives a homomorphism $\mathbb{Z} \oplus \mathbb{Z} \cong \pi_{1}(T) \rightarrow \pi_{1}\left(X_{m}\right)=B_{m}$. Since the basis braids are the images of generators of $\mathbb{Z} \oplus \mathbb{Z}$ by this homomorphism, they are commutative.

(2) For any commutative $m$-braids $a$ and $b$, let us consider a map $f: \mathbf{m} \cup \mathbf{l} \rightarrow X_{m}$ such that the closed paths $\left.f\right|_{\mathbf{m}}$ and $\left.f\right|_{\mathbf{I}}$ in $X_{m}$ represent $a$ and $b$ respectively. Since $a$ and $b$ are commutative, $a b a^{-1} b^{-1}$ is isotopic to the trivial braid, and it follows that the closed path $l$ in $X_{m}$ representing $a b a^{-1} b^{-1}$ is null-homotopic. Hence we can take a 2-disk in $X_{m}$ such that the boundary is $l$ : thus $f$ can be extended to a map 
from $T$ to $X_{m}$. Since $\pi_{2}\left(X_{m}\right)=0$ (see Fadell and Neuwirth [15]), such an extension is unique (up to equivalence). This means that there exists a unique torus-covering $T^{2}$-link with basis braids $a$ and $b$.

By Corollary 2.6, we have the following corollary.

Corollary 2.9 For commutative $m$-braids $a$ and $b$, the following equivalence relations hold:

$$
\mathcal{S}_{m}(a, b) \sim \mathcal{S}_{m}\left(b^{-1}, a\right), \quad \mathcal{S}_{m}(a, b) \sim \mathcal{S}_{m}\left(a, a^{2} b\right)
$$

Proof Let $\Gamma$ be an $m$-chart on $T$ without black vertices and with basis braids $a$ and $b$. By Lemma 2.7 and Lemma $2.8(2), \Gamma$ presents $\mathcal{S}_{m}(a, b)$. By Corollary 2.6, the torus-covering $T^{2}$-links presented by $\Gamma, \rho(\Gamma)$ and $\tau^{2}(\Gamma)$ are equivalent. The basis braids of $\rho(\Gamma)$ are $b^{-1}$ and $a$. Further, the basis braids of $\tau^{2}(\Gamma)$ are $a$ and $a^{2} b$; see Figure 3. Thus we have the required equivalent relations.

Using this corollary, we can for example show the following proposition. We remark the result before the statement of Corollary 5.7. An oriented surface link $S$ is invertible if $S$ is equivalent to its orientation-reversed image $-S$.

Proposition 2.10 For any integers $p$ and $n, \mathcal{S}_{4}\left(\sigma_{1} \sigma_{2}^{p} \sigma_{3}, \Delta^{2 n}\right)$ is invertible, where $\Delta$ is a half-twist of a bundle of 4 parallel strands.

Proof Let us determine the basis braids of $-\mathcal{S}_{m}(a, b)$, as follows. Put $S=\mathcal{S}_{m}(a, b)$. Then $(-S) \cap p_{T}^{-1}(-\mathbf{m})$ is the closure of $-a=\bar{a}^{-1}$, and $(-S) \cap p_{T}^{-1}(-\mathbf{l})$ is the closure of $-b=\bar{b}^{-1}$. Here $-x(x=\mathbf{m}, \mathbf{l}, a, b)$ is the orientation-reversed image of $x$, and $\bar{a}$ (respectively $\bar{b}$ ) is the $m$-braid obtained from $a$ (respectively $b$ ) by replacing $\sigma_{i}$ with $\sigma_{i}^{-1}$ for each standard generator $\sigma_{i}$ of $B_{m}$. Hence $(-S) \cap p_{T}^{-1}(\mathbf{m})$ and $(-S) \cap p_{T_{-}}^{-1}$ (l) are the closures of $\bar{a}$ and $\bar{b}$ respectively; thus the basis braids of $-S$ are $\bar{a}$ and $\bar{b}$. From now on, put $S=\mathcal{S}_{4}\left(\sigma_{1} \sigma_{2}^{p} \sigma_{3}, \Delta^{2 n}\right)$. By the above argument and Lemma 2.8 (2), $-S$ is equivalent to $\mathcal{S}_{4}\left(\sigma_{1}^{-1} \sigma_{2}^{-p} \sigma_{3}^{-1}, \Delta^{-2 n}\right)$. Applying the first equivalent relation of Corollary 2.9 twice, we have $\mathcal{S}_{m}(a, b) \sim \mathcal{S}_{m}\left(b^{-1}, a\right) \sim$ $\mathcal{S}_{m}\left(a^{-1}, b^{-1}\right)$. Thus $-S \sim \mathcal{S}_{4}\left(\sigma_{3} \sigma_{2}^{p} \sigma_{1}, \Delta^{2 n}\right)$. Regarding the $i-$ th string of the basis braids as the $(4-i)$-th string $(i=1, \ldots, 4)$, we can regard the basis braids as $\sigma_{1} \sigma_{2}^{p} \sigma_{3}$ and $\Delta^{2 n}$ respectively; thus $-S \sim \mathcal{S}_{4}\left(\sigma_{1} \sigma_{2}^{p} \sigma_{3}, \Delta^{2 n}\right)=S$.

We show that some torus-covering $T^{2}$-links are equivalent to known $T^{2}-$ links. Let $b$ an $m$-braid, and let $Q_{m}$ be the starting point set of $b$. Let us denote by $\hat{b}$ the closure of $b$. 
Proposition 2.11 The torus-covering $T^{2}-\operatorname{link} \mathcal{S}_{m}(b, e)$ is equivalent to the spun $T^{2}-$ link of $\hat{b}$.

The 4-space $\mathbb{R}^{4}$ is constructed by rotating the upper half plane $\mathbb{R}_{+}^{3}=\mathbb{R}^{2} \times[0, \infty)$ in $\mathbb{R}^{2} \times \mathbb{R}^{2}$ around the axis $\mathbb{R}^{2} \times\{0\}$. This structure is called an open book structure. Let $B^{3}$ be a 3-ball in $\mathbb{R}_{+}^{3}$, and let us naturally identify the orbit of $B^{3}$ with $B^{3} \times S^{1}$ in $\mathbb{R}^{4}$, where $S^{1}$ is a circle. Let $\pi: B^{3} \times S^{1} \rightarrow B^{3}$ be the projection. A surface link $S$ in $B^{3} \times S^{1}$ can be considered as $\bigcup_{t \in S^{1}} S_{t} \times\{t\}$, where $S_{t}=\pi\left(S \cap\left(B^{3} \times\{t\}\right)\right) \subset B^{3}$. We call the collection $\left\{S_{t}\right\}_{t \in S^{1}}$ the motion picture of $S$ along $S^{1}$.

Let $L$ be a classical link in $B^{3}$. The spun $T^{2}$-link of $L$ is the surface link defined by the motion picture $S_{t}=L$ for $t \in S^{1}$ (see Livingston [26] and Boyle [7; 8]).

In an open book structure of $\mathbb{R}^{4}$, we naturally identify the orbit of $B^{3}$ with $B^{3} \times S^{1}$. Let us consider un unknotted circle $S^{1} \subset B^{3}$ with the base point $x_{0}$. The orbit of this $S^{1}$ is a standard torus. We identify it with $T$, by identifying $S^{1} \times\{0\} \subset B^{3} \times\{0\}$ with the meridian $\mathbf{m}$, and $\left\{x_{0}\right\} \times S^{1} \subset B^{3} \times S^{1}$ with the longitude $\mathbf{l}$. Further, we identify $N(T)$ with the orbit of the unknotted solid torus $N(\mathbf{m})=\pi\left(p_{T}^{-1}(\mathbf{m})\right)$ in $B^{3}$. Put $D\left(x_{0}\right)=\pi\left(p_{T}^{-1}\left(x_{0}\right)\right)$. Let us identify $S^{1}$ with $[0,1] / \sim$, where $0 \sim 1$. Let us assume that the closure $\hat{b}$ is in the solid torus $N(\mathbf{m})$ such that the identified corresponding ends are in $D\left(x_{0}\right)$. Further we regard a braided surface over $T$ as in $N(T) \subset \mathbb{R}^{4}$.

Proof of Proposition 2.11 Let $S$ be the surface defined by the motion picture $S_{t}=\widehat{b}$ for $t \in S^{1}$. By definition, $S$ is the spun $T^{2}$-link of $\hat{b}$. Since $S$ is a braided surface over $T$ with no branch points, $S$ is a torus-covering $T^{2}$-link. Let us determine the basis braids. Since $\pi\left(S \cap p_{T}^{-1}(\mathbf{m})\right)=S_{0} \cap N(\mathbf{m})=\hat{b}$, one basis braid is $b$. By definition, we have $S \cap p_{T}^{-1}(\mathbf{l})=\bigcup_{t \in[0,1]}\left(S_{t} \cap D\left(x_{0}\right)\right) \times\{t\}$. Since $S_{t} \cap D\left(x_{0}\right)=Q_{m}$ for any $t, S \cap p_{T}^{-1}(\mathbf{I})$ is the closure of the trivial $m$-braid $e=Q_{m} \times[0,1]$. Thus $S$ is a torus-covering $T^{2}$-link with basis $m$-braids $b$ and $e$, and it follows from Lemma 2.8 (2) that $S$ is equivalent to $\mathcal{S}_{m}(b, e)$.

Let us identify the 3-ball $B^{3}$ with the unit ball in the $x y z$-space. Let us rotate a classical link $L$ in $B^{3}$ around the $z$-axis once, and identify the resulting link with the original link. The orbit of $L$ forms a surface link, called the turned spun $T^{2}$-link of $L$ (see Livingston [26] and Boyle [8]).

Proposition 2.12 The torus-covering $T^{2}$-link $\mathcal{S}_{m}(b, b)$ is equivalent to the turned spun $T^{2}-$ link of $\hat{b}$. 
Proof We can assume that the solid torus $N(\mathbf{m}) \subset B^{3}$ is fixed as a set when we rotate it around the $z$-axis. Let $\left\{h_{u}\right\}_{u \in[0,1]}$ be an isotopy of $B^{3}$ which describes the rotation of the solid torus $N(\mathbf{m})$ around the $z$-axis once. Let $S$ be the surface defined by the motion picture $S_{t}=\bigcup_{u \in[0,1]} h_{t}(\hat{b})$ for $t \in S^{1}$. By definition, $S$ is the turned spun $T^{2}$-link of $\hat{b}$. By the same argument with the proof of Proposition 2.11, $S$ is a torus-covering $T^{2}$-link with $S \cap p_{T}^{-1}(\mathbf{m})=\hat{b}$. Regarding $N(\mathbf{m})$ as $D^{2} \times S^{1}$ such that $D\left(x_{0}\right)=D^{2} \times\{0\}$, we have $S_{t} \cap D\left(x_{0}\right)=h_{t}(\hat{b}) \cap\left(D^{2} \times\{0\}\right)=p\left(\hat{b} \cap\left(D^{2} \times\{t\}\right)\right) \times\{0\}$ for $t \in[0,1]$, where $p: D^{2} \times S^{1} \rightarrow D^{2}$ is the projection; thus $S \cap p_{T}^{-1}(\mathbf{l})=$ $\bigcup_{t \in[0,1]}\left(S_{t} \cap D\left(x_{0}\right)\right) \times\{t\}=\hat{b}$. Thus the basis braids of $S$ are $b$ and $b$, and $S \sim \mathcal{S}_{m}(b, b)$ by Lemma $2.8(2)$.

Let $L$ be a classical link in $B^{3}$ such that rotating $L$ around the $z$-axis by $2 k \pi / n$ results in the original $L$ as a set. Then let us rotate $L$ around the $z$-axis by $2 k \pi / n$, and identify the resulting link with the original link. The orbit of $L$ forms a surface link, called a symmetry-spun $T^{2}$-link (see Teragaito [31]).

Let $b^{n}$ be an $m$-braid in $D^{2} \times[0,1]$ such that $b^{n} \cap\left(D^{2} \times I_{j}\right)=b$, where $I_{j}=$ $[(j-1) / n, j / n](j=1,2, \ldots, n)$.

Proposition 2.13 The torus-covering $T^{2}$-link $\mathcal{S}_{m}\left(b^{n}, b^{k}\right)$ is equivalent to a symmetryspun $T^{2}-$ link, which is constructed by turning $\widehat{b^{n}}$ by $2 k \pi / n$ around the axis while spinning.

By Teragaito [31, Theorem 8], the symmetry-spun $T^{2}$-link $\mathcal{S}_{m}\left(b^{n}, b^{k}\right)$ is equivalent to either $\mathcal{S}_{m}\left(b^{r}, e\right)$ or $\mathcal{S}_{m}\left(b^{r}, b^{r}\right)$, where $r=\operatorname{gcd}(n, k)$. This can be shown by Corollary 2.9, too.

Proof Let $\left\{h_{u}\right\}_{u \in[0,1]}$ be an isotopy of $B^{3}$ which describes the rotation of the solid torus $N(\mathbf{m})$ around the axis by $2 k \pi / n$. Let $S$ be the surface defined by the motion picture $S_{t}=\bigcup_{u \in[0,1]} h_{t}(\hat{b})$ for $t \in S^{1}$. By definition, $S$ is the symmetry-spun $T^{2}-$ link in question. By the same argument with the proof of Proposition 2.11, we can see that $S$ is a torus-covering $T^{2}$-link with $S \cap p_{T}^{-1}(\mathbf{m})=\widehat{b^{n}}$. Using the same notation with the proof of Proposition 2.12, we have $S_{t} \cap D\left(x_{0}\right)=h_{t}\left(\widehat{b^{n}}\right) \cap\left(D^{2} \times\{0\}\right)=$ $p\left(\widehat{b^{n}} \cap\left(D^{2} \times\{k t / n\}\right)\right) \times\{0\}$ for $t \in S^{1}$. So $S \cap p_{T}^{-1}(\mathbf{I})=\bigcup_{t \in S^{1}}\left(S_{t} \cap D\left(x_{0}\right)\right) \times\{t\}=\widehat{b^{k}}$. Thus the basis braids of $S$ are $b^{n}$ and $b^{k}$, and $S \sim \mathcal{S}_{m}\left(b^{n}, b^{k}\right)$ by Lemma 2.8 (2).

Let us call an edge of an $m$-chart a loop if it is connected with no vertices. Let us consider an $m$-chart on $T$ with no vertices.

Proposition 2.14 An $m$-chart on $T$ with no vertices presents a spun $T^{2}$-link or a turned spun $T^{2}-$ link. 
Proof By Lemma 2.7, an $m$-chart with no vertices presents a torus-covering $T^{2}$-link. Let us determine the basis braids, as follows. An $m$-chart with no vertices consists of a finite number of loops. By Lemma 2.15, we can assume that any loop does not bound a 2-disk in $T$. Then, by an ambient isotopy of $T$, we can make all the loops parallel and moreover geodesic. The $m$-chart is as in Figure 4; thus the basis braids

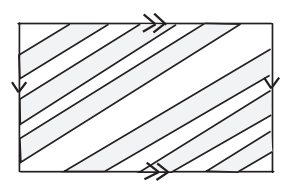

Figure 4

presented by $\Gamma$ are $b^{n}$ and $b^{k}$, for an $m$-braid $b$ and integers $n$ and $k$. Its presenting torus-covering $T^{2}$-link is equivalent to a symmetry-spun $T^{2}$-link by Proposition 2.13; thus it is equivalent to either a spun $T^{2}$-link or a turned spun $T^{2}-$ link.

Lemma 2.15 An $m$-chart on $T$ with no vertices is $C$-move equivalent to an $m$-chart such that each loop does not bound a 2-disk in $T$.

Proof For a 2-disk $D$ in $T$ such that $\partial D$ intersects an $m$-chart $\Gamma$ transversely, if there are no black vertices in $\Gamma \cap D$, then by a CI-move we can redraw the $m$-chart within $D$ as we like as long as it has no black vertices (see Kamada [24]). Hence, if there is a loop which bounds a 2-disk in $T$, then we can remove it by applying a CI-move around the neighborhood of the loop.

Proposition 2.14 can be extended to an $m$-chart on $T$ with neither black nor white vertices, as follows. The split union of two surface links $S_{1}$ and $S_{2}$ is a surface link presented by the union of the copies of $S_{1}$ and $S_{2}$ such that for a 3-sphere $S^{3}$ embedded in $\mathbb{R}^{4}, S_{1}$ is inside of $S^{3}$ and $S_{2}$ is outside. The 3 -sphere $S^{3}$ is called a separating 3-sphere.

Theorem 2.16 An $m$-chart on $T$ with neither black nor white vertices presents either a spun $T^{2}$-link, a turned spun $T^{2}$-link or the split union of spun $T^{2}$-links and turned spun $T^{2}-$ links.

Let $\Gamma$ be an $m$-chart with neither black nor white vertices. Then every vertex of $\Gamma$ is of degree 4 . Since the diagonal edges around a vertex of degree 4 have the same label and coherent orientation (see Figure 1), we can regard the union of connected edges of $\Gamma$ with the label $i$ as an oriented immersed circle with the label $i$. Let us call it just an immersed circle. Since the edges around a vertex of degree 4 have the 
labels $i$ and $j$ with $|i-j|>1$, we can regard $\Gamma$ as consisting of immersed circles with transverse intersections such that each intersection is formed by two immersed circles with the labels $i$ and $j$ with $|i-j|>1$.

Proof Let $\Gamma$ be an $m$-chart with neither black nor white vertices. Let $i>0$ be the minimum integer which does not appear as a label of $\Gamma$. Let $\Gamma_{<i}$ be a subgraph in $T$ consisting of the edges of $\Gamma$ with the labels smaller than $i$. Further, attach to each edge of this $\Gamma_{<i}$ the orientation and label induced from $\Gamma$. Since $\Gamma$ can be regarded as consisting of immersed circles, so can $\Gamma_{<i}$; thus $\Gamma_{<i}$ presents a new $m$-chart on $T$ with neither black nor white vertices. Similarly, let $\Gamma_{>i}$ be a subgraph in $T$ consisting of the edges of $\Gamma$ with the labels larger than $i$, with induced labels and orientations. Then $\Gamma_{>i}$ also presents a new $m$-chart on $T$ with neither black nor white vertices. Since $\Gamma$ has no edge with the label $i, \Gamma=\Gamma_{<i} \cup \Gamma_{<i}$. By Lemma 2.17, the torus-covering link presented by $\Gamma$ is equivalent to the split union of two torus-covering links presented by $\Gamma_{<i}$ and $\Gamma_{>i}$.

If there is an immersed circle which bounds a 2 -disk in $T$, then we can remove it by applying a CI-move by the same argument of Lemma 2.15. Thus, taking new $i$ if necessary, we can assume that $\Gamma_{<i}$ satisfies the conditions of Lemma 2.18, ie (1) any immersed circle does not bound a 2 -disk in $T$, and (2) there is at least one immersed circle with the label $j$ for every label $j<i$. Thus $\Gamma_{<i}$ presents a spun $T^{2}$-link or a turned spun $T^{2}$-link by Lemma 2.18. Using induction for $i$, we can see that $\Gamma$ presents a spun $T^{2}-$ link, a turned spun $T^{2}$-link or the split union of spun $T^{2}$-links and turned spun $T^{2}-$ links.

Lemma 2.17 The torus-covering link presented by $\Gamma_{<i} \cup \Gamma_{>i}$ is equivalent to the split union of two torus-covering links presented by $\Gamma_{<i}$ and $\Gamma_{>i}$.

Proof Let us denote by $S, S_{1}$ and $S_{2}$ the torus-covering links presented by $\Gamma_{<i} \cup \Gamma_{>i}$, $\Gamma_{<i}$ and $\Gamma_{>i}$ respectively. Consider the open book decomposition of $\mathbb{R}^{4}$ as in Proposition 2.11. Let $N_{1}$ (respectively $N_{2}$ ) be a solid torus in $N(\mathbf{m})$ which contains the $j$-th starting point of the basis braids of $S$ for every $j<i$ (respectively $j>i$ ). Since there are no edges of $\Gamma_{<i} \cup \Gamma_{>i}$ with the label $i$, we can assume that $S$ is in the orbit $\left(N_{1} \cup N_{2}\right) \times S^{1}$ such that $S_{1}$ and $S_{2}$ are contained in $N_{1} \times S^{1}$ and $N_{2} \times S^{1}$ respectively. Let us take a 2-disk in $\mathbb{R}_{+}^{3}$ as in Figure 5. The orbit of this 2-disk forms a separating 3-sphere. Thus $S$ is the split union of $S_{1}$ and $S_{2}$.

Lemma 2.18 Let $\Gamma$ be an $m$-chart on $T$ with neither black nor white vertices satisfying (1) any immersed circle does not bound a 2-disk in $T$, and (2) there is at least one immersed circle with the label $j$ for every label $j$. Then $\Gamma$ presents either a spun $T^{2}$-link or a turned spun $T^{2}-$ link. 


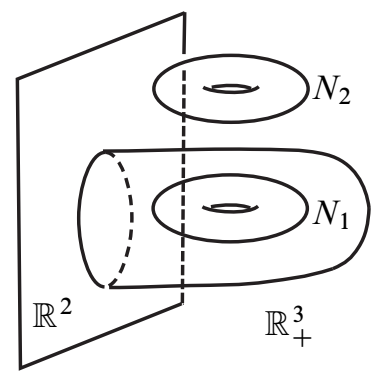

Figure 5

Proof By the definition of an $m$-chart, there are no intersections between the immersed circles with the labels $j-1$ and $j$. Thus, together with (1), the immersed circles with the labels $j-1$ and $j$ can be made parallel by an isotopy of $T$. Hence it follows from (2) that each oriented immersed circle goes $n$ times around the meridian $\mathbf{m}$ and $k$ times around the longitude $\mathbf{l}$, or $-n$ times around $\mathbf{m}$ and $--k$ times around $\mathbf{l}$, for some fixed integers $n$ and $k$. Since there are only intersections of immersed circles with the labels $i$ and $j$ with $|i-j|>1$ as vertices of $\Gamma$, we can remove all the vertices by CI-moves of type (4) (see Figure 2). Then the $m$-chart $\Gamma$ presents either a spun $T^{2}$-link or a turned spun $T^{2}$-link by Proposition 2.14 .

We showed in Theorem 2.16 that an $m$-chart on $T$ with neither black nor white vertices presents either a spun $T^{2}$-link, a turned spun $T^{2}$-link or the split union of spun $T^{2}$-links and turned spun $T^{2}$-links. We will show in Theorem 3.12 that there is a torus-covering $T^{2}$-link which is not equivalent to either a spun $T^{2}-$ link, a turned spun $T^{2}$-link or the split union of spun $T^{2}$-links and turned spun $T^{2}-$ links. Its presenting $m$-chart on $T$ does not have black vertices (Lemma 2.7) but does have white vertices (Corollary 4.3 (2)).

\section{Knot groups and link groups}

From now on in this paper, we consider torus-covering $T^{2}$-links. By Lemma 2.8, the basis braids of a torus-covering $T^{2}$-link are commutative, and for any commutative $m$-braids $a$ and $b$, there exists a unique torus-covering $T^{2}$-link with basis braids $a$ and $b$. In this section, first we compute the link group of $\mathcal{S}_{m}(a, b)$ (Proposition 3.1). Using this proposition, we will show that a certain 2-component torus-covering $T^{2}-$ link has a nonclassical link group (Theorem 3.2). We show its knot version as well: a certain torus-covering $T^{2}$-knot has a nonclassical knot group (Theorem 3.7). Further we show that the torus-covering $T^{2}$-link of Theorems 3.2 or 3.7 is not equivalent to 
either a spun $T^{2}-$ link, a turned spun $T^{2}-$ link or the split union of spun $T^{2}-$ links and turned spun $T^{2}$-links (Theorem 3.12).

Before the statement of Proposition 3.1, we will give the definition of Artin's automorphism (see Kamada [24]). Let $b$ be an $m$-braid in a cylinder $D^{2} \times[0,1]$, and let $Q_{m}$ be the starting point set of $b$. Let $\left\{h_{u}\right\}_{u \in[0,1]}$ be an isotopy of $D^{2}$ rel $\partial D^{2}$ such that $\bigcup_{u \in[0,1]} h_{u}\left(Q_{m}\right) \times\{u\}=b$. Let $\mathcal{A}^{b}:\left(D^{2}, Q_{m}\right) \rightarrow\left(D^{2}, Q_{m}\right)$ be the terminal map $h_{1}$, and consider the induced map $\mathcal{A}_{*}^{b}: \pi_{1}\left(D^{2}-Q_{m}\right) \rightarrow \pi_{1}\left(D^{2}-Q_{m}\right)$. It is known (see Artin [1]) that $\mathcal{A}^{b}$ is uniquely determined from $b$. We call $\mathcal{A}_{*}^{b}$ Artin's automorphism associated with $b$. Note that $\pi_{1}\left(D^{2}-Q_{m}\right)$ is naturally isomorphic to the free group $F_{m}$ generated by the standard generators $x_{1}, x_{2}, \ldots, x_{m}$ of $\pi_{1}\left(D^{2}-Q_{m}\right)$. By $\mathcal{A}_{*}^{b}$, the braid group $B_{m}$ acts on $\pi_{1}\left(D^{2}-Q_{m}\right)$. It is presented by

and

$$
\mathcal{A}_{*}^{\sigma_{i}}\left(x_{j}\right)= \begin{cases}x_{j} x_{j+1} x_{j}^{-1} & \text { if } j=i, \\ x_{j-1} & \text { if } j=i+1, \\ x_{j} & \text { otherwise, }\end{cases}
$$

$$
\mathcal{A}_{*}^{\sigma_{i}^{-1}}\left(x_{j}\right)= \begin{cases}x_{j+1} & \text { if } j=i, \\ x_{j}^{-1} x_{j-1} x_{j} & \text { if } j=i+1, \\ x_{j} & \text { otherwise, }\end{cases}
$$

where $i=1,2, \ldots, m-1$ and $j=1,2, \ldots, m$.

Proposition 3.1 For commutative $m$-braids $a$ and $b$, the link group of $\mathcal{S}_{m}(a, b)$ is presented by:

$$
\left.\pi_{1}\left(\mathbb{R}^{4}-\mathcal{S}_{m}(a, b)\right)=\left\langle x_{1}, \ldots, x_{m}\right| x_{j}=\mathcal{A}_{*}^{a}\left(x_{j}\right)=\mathcal{A}_{*}^{b}\left(x_{j}\right) \text { for } j=1,2, \ldots, m\right\rangle
$$

Proof Put $S=\mathcal{S}_{m}(a, b)$. Let $Q_{m}$ be a set of $m$ distinct interior points of $D^{2}$, and let $q_{0}$ be a point of $\partial D^{2}$. The space $N(T)-S$ is a fiber bundle over $T$ with the fiber $D^{2}-Q_{m}$ whose monodromy is given by $\mathcal{A}^{a}$ and $\mathcal{A}^{b}$. Let us take commutative $\mathcal{A}^{a}$ and $\mathcal{A}^{b}$. Then we have

$$
\begin{aligned}
N(T)-S & \cong\left(\left(D^{2}-Q_{m}\right) \times I \times I\right) /(x, 0, u) \sim\left(\mathcal{A}^{a}(x), 1, u\right),(x, u, 0) \sim\left(\mathcal{A}^{b}(x), u, 1\right) \\
& =M \times I /(x, u, 0) \sim\left(\mathcal{A}^{b}(x), u, 1\right)
\end{aligned}
$$

where

$$
M=\left(\left(D^{2}-Q_{m}\right) \times I\right) /(x, 0) \sim\left(\mathcal{A}^{a}(x), 1\right)
$$

and $x \in D^{2}-Q_{m}$ and $u \in I$.

We compute $\pi_{1}(M)$, as follows. Since $M$ is a mapping torus whose monodromy is given by $\mathcal{A}^{a}$, by van Kampen's theorem, we can see that $\pi_{1}(M)$ has a presentation 
obtained from $\pi_{1}\left(D^{2}-Q_{m}\right) * \mathbb{Z}$ by adding relations $s^{-1} x s=\mathcal{A}_{*}^{a}(x)$, where $x \in$ $\pi_{1}\left(D^{2}-Q_{m}\right)$, and $s$ is the generator of $\mathbb{Z}$, which is represented by the loop $\left\{q_{0}\right\} \times S^{1}$ with the base point $q_{0}$. Since $\pi_{1}\left(D^{2}-Q_{m}\right)$ is a free group generated by the standard generators $x_{1}, x_{2}, \ldots, x_{m}$ with the base point $q_{0}$, we have:

$$
\left.\pi_{1}(M)=\left\langle x_{1}, x_{2}, \ldots, x_{m}, s\right| s^{-1} x_{j} s=\mathcal{A}_{*}^{a}\left(x_{j}\right) \text { for } j=1,2, \ldots, m\right\rangle
$$

We compute $\pi_{1}(N(T)-S)$, as follows. Since $N(T)-S=M \times I /(x, u, 0) \sim$ $\left(\mathcal{A}^{b}(x), u, 1\right)$, where $x \in D^{2}-Q_{m}$ and $u \in I$ with $(x, u) \in M, N(T)-S$ is a mapping torus whose monodromy is given by $\mathcal{A}^{b} \times \mathrm{id}$. Thus we can see that $\pi_{1}(N(T)-S)$ has a presentation obtained from $\pi_{1}(M) * \mathbb{Z}$ by adding relations $t^{-1} y t=\left(\mathcal{A}_{*}^{b} \times \mathrm{id}_{*}\right)(y)$, where $y \in \pi_{1}(M)$ and $t$ is the generator of $\mathbb{Z}$. Hence together with (3-1), we can see that $\pi_{1}(N(T)-S)$ is presented by

(3-2) $\pi_{1}(N(T)-S)=\left\langle\begin{array}{l|l}x_{1}, x_{2}, \ldots, x_{m}, s, t & \begin{array}{l}s^{-1} x_{j} s=\mathcal{A}_{*}^{a}\left(x_{j}\right), t^{-1} x_{j} t=\mathcal{A}_{*}^{b}\left(x_{j}\right), \\ t^{-1} s t=s, \text { for } j=1,2, \ldots, m\end{array}\end{array}\right\}$

where $s$ and $t$ are represented by the loops $\mathbf{m}$ and $\mathbf{l}$ respectively.

We compute $\pi_{1}\left(S^{4}-S\right)$, as follows. We have $S^{4}-S=(N(T)-S) \cup_{\partial N(T)} E^{4}$. The fundamental group $\pi_{1}(N(T)-S)$ has the presentation (3-2). We obtain $\pi_{1}\left(E^{4}\right)$, as follows. Since $N(T)$ is a tubular neighborhood of $T$, and $T$ is the boundary of the standard unknotted solid torus in $\mathbb{R}^{3} \times\{0\}$, we can see that the fundamental group of $E^{4}=\operatorname{cl}\left(S^{4}-N(T)\right)$ is the knot group of a trivial torus knot. Hence $\pi_{1}\left(E^{4}\right)$ is an infinite cyclic group, where the generator $r$ is represented by the loop $\mathbf{r}$ (see Carter and Saito [14, Section 5.2]). Next we obtain $\pi_{1}\left(\partial E^{4}\right)$, as follows. Since $\partial E^{4}=\partial N(T)=\partial D^{2} \times T$ is a 3-dimensional torus $S^{1} \times S^{1} \times S^{1}, \pi_{1}(\partial N(T))$ is isomorphic to $\mathbb{Z} \oplus \mathbb{Z} \oplus \mathbb{Z}$, where the generators $r^{\prime}, m^{\prime}$ and $l^{\prime}$ are represented by the loops $\mathbf{r}, \mathbf{m}$ and $\mathbf{l}$ respectively. Let $i_{1}: \partial N(T) \rightarrow N(T)-S$ and $i_{2}: \partial N(T) \rightarrow E^{4}$ be inclusion maps. Since $i_{1 *}\left(r^{\prime}\right)=x_{1} x_{2} \cdots x_{m}, i_{1 *}\left(m^{\prime}\right)=s$ and $i_{1 *}\left(l^{\prime}\right)=t$ in $\pi_{1}(N(T)-S)$, and $i_{2 *}\left(r^{\prime}\right)=r, i_{2 *}\left(m^{\prime}\right)=1$ and $i_{2 *}\left(l^{\prime}\right)=1$ in $\pi_{1}\left(E^{4}\right)$, by van Kampen's theorem $\pi_{1}\left(S^{4}-S\right)=\pi_{1}\left((N(T)-S) \cup_{\partial N(T)} E^{4}\right)$ is presented by

$$
\left\langle\begin{array}{l|l}
x_{1}, \ldots, x_{m}, s, t, r & \begin{array}{l}
s^{-1} x_{j} s=\mathcal{A}_{*}^{a}\left(x_{j}\right), t^{-1} x_{j} t=\mathcal{A}_{*}^{b}\left(x_{j}\right), t^{-1} s t=s, \\
r=x_{1} x_{2} \cdots x_{m}, s=1, t=1, \text { for } j=1,2, \ldots, m
\end{array}
\end{array}\right\}
$$

which is the required formula.

Theorem 3.2 Put $S_{n}=\mathcal{S}_{4}\left(\sigma_{1} \sigma_{3}, \Delta^{2 n}\right)$, where $\Delta=\sigma_{1} \sigma_{2} \sigma_{3} \sigma_{1} \sigma_{2} \sigma_{1}$ (see Figure 6) and $n$ is a positive integer. Then the link group of $S_{n}$ is not a classical link group. Moreover, $S_{n}$ and $S_{m}$ are not equivalent for $n \neq m$. 


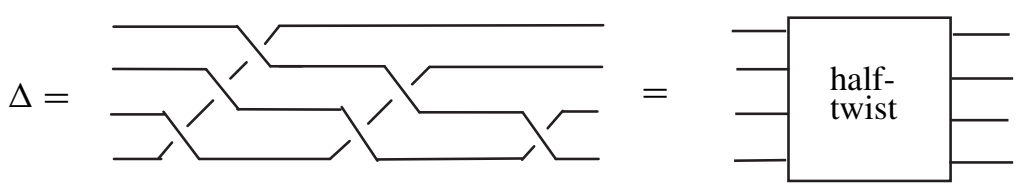

Figure 6: A half-twist $\Delta$

Since $\Delta^{2 n}$ is a central element of $B_{4}$ and the closure of $\sigma_{1} \sigma_{3}$ is a classical link with two components, the torus-covering $T^{2}$-link $S_{n}$ has two components. Each component of $S_{n}$ is equivalent to $\mathcal{S}_{2}\left(\sigma_{1}, \sigma_{1}^{2 n}\right)$, which is equivalent to $\mathcal{S}_{2}\left(\sigma_{1}, e\right)$ by Corollary 2.9. By Proposition 2.11, it is equivalent to the spun $T^{2}$-knot of $\widehat{\sigma_{1}}$. Since $\widehat{\sigma_{1}}$ is a trivial knot, this is an unknotted $T^{2}$-knot. Thus each component of $S_{n}$ is an unknotted $T^{2}$-knot.

Proof By Proposition 3.1, the link group $G_{n}$ of $S_{n}$ is computed as follows. Let $x_{1}, \ldots, x_{4}$ be the generators. The relations concerning the basis braid $\sigma_{1} \sigma_{3}$ are $x_{1}=x_{2}$ and $x_{3}=x_{4}$. The other relations concerning the basis braid $\Delta^{2 n}$ are:

$$
\begin{aligned}
& x_{1}=\left(x_{1} x_{2} x_{3} x_{4}\right)^{n} x_{1}\left(x_{1} x_{2} x_{3} x_{4}\right)^{-n} \\
& x_{2}=\left(x_{1} x_{2} x_{3} x_{4}\right)^{n} x_{2}\left(x_{1} x_{2} x_{3} x_{4}\right)^{-n} \\
& x_{3}=\left(x_{1} x_{2} x_{3} x_{4}\right)^{n} x_{3}\left(x_{1} x_{2} x_{3} x_{4}\right)^{-n} \\
& x_{4}=\left(x_{1} x_{2} x_{3} x_{4}\right)^{n} x_{4}\left(x_{1} x_{2} x_{3} x_{4}\right)^{-n}
\end{aligned}
$$

Putting $a=x_{1}=x_{2}$ and $b=x_{3}=x_{4}$, we have:

$$
G_{n}=\left\langle a, b \mid\left(a^{2} b^{2}\right)^{n} b=b\left(a^{2} b^{2}\right)^{n},\left(a^{2} b^{2}\right)^{n} a=a\left(a^{2} b^{2}\right)^{n}\right\rangle
$$

By Lemma 3.4, $G_{1}$ is not a classical link group. Let us consider the case for $n>1$. For $n>1$, let $Z_{n}$ be the subgroup of $G_{n}$ generated by $h_{n}=\left(a^{2} b^{2}\right)^{n}$. By Lemma 3.5, $Z_{n}$ is the center of $G_{n}$ for $n>1$. Further, $Z_{n}$ is an infinite cyclic group; thus the center of $G_{n}$ is nontrivial.

We will show that $G_{n}(n>1)$ is not a classical link group. Since the torus-covering $T^{2}-$ link $S_{n}$ consists of two components, we show that $G_{n}$ is not a classical 2-component link group, as follows. It is known (see Burde and Murasugi [9]) that if the center of the group of a classical 2-component link $L$ is nontrivial, then the link group of $L$ is isomorphic to one of the groups of type (a), (b) or (c) as follows:

(a) $Z \times \mathbb{Z}$,

(b) $(Z \times \mathbb{Z}) * Z \mathbb{Z}$,

(c) $(Z \times \mathbb{Z}) * Z(\mathbb{Z} * \mathbb{Z} \mathbb{Z})$ 
where $\mathbb{Z}$ is an infinite cyclic group, and $Z=\langle h\rangle$ is a "special" infinite cyclic group which is the center of the link group of types (b) and (c). In type (b), the amalgamation concerning the last factor $\mathbb{Z}=\langle q\rangle$ is given by $h=q^{\alpha}$ for an integer $\alpha>1$. In type (c), the last factor $\mathbb{Z} * \mathbb{Z} \mathbb{Z}$ is the group of the torus $(\alpha, \beta)$-knot, ie $\mathbb{Z} * \mathbb{Z} Z=\left\langle x, y \mid x^{\alpha}=y^{\beta}\right\rangle$ for coprime positive integers $\alpha$ and $\beta$, and the amalgamation is given by $h=x^{\alpha}=y^{\beta}$.

Since an infinite cyclic group $Z_{n}$ is the center of $G_{n}$ by Lemma 3.5, it suffices to show that $G_{n}(n>1)$ is neither of type (b) nor (c). Further, in these cases, the center $Z$ equals $Z_{n}$ and $h=h_{n}$.

Case (b) If $G_{n}$ is of type (b), then $G_{n}=\left(Z_{n} \times \mathbb{Z}\right) * Z_{n} \mathbb{Z}=\left(\left\langle h_{n}\right\rangle \times\langle k\rangle\right) * Z_{n}\langle q\rangle$, where the amalgamation is given by $h_{n}=q^{\alpha}$ for an integer $\alpha>1$. Put $h_{n}^{\prime}=f\left(h_{n}\right)$ and $q^{\prime}=f(q)$ for a natural epimorphism

$$
f: G_{n} \rightarrow \mathbb{Z} / 2 \mathbb{Z} * \mathbb{Z} / 2 \mathbb{Z}=\left\langle a^{\prime}\right\rangle *\left\langle b^{\prime}\right\rangle
$$

where $a^{\prime}=f(a)$ and $b^{\prime}=f(b)$, which are the basis. Since $h_{n}=\left(a^{2} b^{2}\right)^{n}$, we see that $h_{n}^{\prime}=1$. Since $h_{n}=q^{\alpha}$, it follows that $q^{\prime \alpha}=1$. If $q^{\prime}=1$, then $f\left(G_{n}\right)=\langle f(k)\rangle$, which is generated by at most one generator. However, $f\left(G_{n}\right)$ is generated by two generators $a^{\prime}$ and $b^{\prime}$. Hence $q^{\prime}$ is nontrivial. Since $\alpha>1$ and a nontrivial element of $f\left(G_{n}\right)=\mathbb{Z} / 2 \mathbb{Z} * \mathbb{Z} / 2 \mathbb{Z}$ has order 2 or $\infty$ by Lemma $3.6, q^{\prime}$ has order 2 ; thus $\alpha=2$. Consider the abelianization map

$$
\phi: G_{n} \rightarrow G_{n} /\left[G_{n}, G_{n}\right]=\mathbb{Z} \times \mathbb{Z}
$$

and put $\bar{a}=\phi(a)$ and $\bar{b}=\phi(b)$, which are the basis. Since $h_{n}=q^{2}$ and $\phi\left(h_{n}\right)=$ $\bar{a}^{2 n} \bar{b}^{2 n}$, it follows that $\phi(q)=\bar{a}^{n} \bar{b}^{n}$. Consider the abelianization map

$$
\phi^{\prime}: f\left(G_{n}\right)=\mathbb{Z} / 2 \mathbb{Z} * \mathbb{Z} / 2 \mathbb{Z} \rightarrow \mathbb{Z} / 2 \mathbb{Z} \times \mathbb{Z} / 2 \mathbb{Z}
$$

and put $\bar{a}^{\prime}=\phi^{\prime}\left(a^{\prime}\right)$ and $\bar{b}^{\prime}=\phi^{\prime}\left(b^{\prime}\right)$, which are the basis. Since $\phi(q)=\bar{a}^{n} \bar{b}^{n}$, it follows that $\phi^{\prime}\left(q^{\prime}\right)=1$ if $n$ is even, and $\phi^{\prime}\left(q^{\prime}\right)=\bar{a}^{\prime} \bar{b}^{\prime}$ if $n$ is odd; thus $\phi^{\prime}\left(q^{\prime}\right)=1$ or $\bar{a}^{\prime} \bar{b}^{\prime}$. However, $\phi^{\prime}\left(q^{\prime}\right)=\bar{a}^{\prime}$ or $\bar{b}^{\prime}$, as follows. Since $h_{n}=q^{2}$, it follows that $q^{\prime 2}=1$; thus $q^{\prime}=\xi^{-1} a^{\prime} \xi$ or $\xi^{-1} b^{\prime} \xi$ for some $\xi \in f\left(G_{n}\right)$ by Lemma 3.6. Thus $\phi^{\prime}\left(q^{\prime}\right)=\bar{a}^{\prime}$ or $\bar{b}^{\prime}$. This is a contradiction.

Case (c) If $G_{n}$ is of type (c), then $G_{n}=\left(Z_{n} \times \mathbb{Z}\right) * Z_{n}(\mathbb{Z} * \mathbb{Z})$, where $\mathbb{Z} * \mathbb{Z}=$ $\left\langle x, y \mid x^{\alpha}=y^{\beta}\right\rangle$ for coprime positive integers $\alpha$ and $\beta$, and the amalgamation is given by $h_{n}=x^{\alpha}=y^{\beta}$. Since $h_{n}=\left(a^{2} b^{2}\right)^{n}$, we see that $h_{n}^{\prime}=1$, where $h_{n}^{\prime}=f\left(h_{n}\right)$. Since $h_{n}=x^{\alpha}=y^{\beta}$, it follows that $x^{\prime \alpha}=y^{\prime \beta}=1$, where $x^{\prime}=f(x)$ and $y^{\prime}=f(y)$. If $x^{\prime}=1$ and $y^{\prime}=1$, then $f\left(\left\langle x, y \mid x^{\alpha}=y^{\beta}\right\rangle\right)=1$ and it follows that $f\left(G_{n}\right)$ is generated by at most one generator. However, $f\left(G_{n}\right)$ is generated by two generators $a^{\prime}$ and $b^{\prime}$. Hence we can assume that $x^{\prime}$ is nontrivial. Since any element of $f\left(G_{n}\right)=\mathbb{Z} / 2 \mathbb{Z} * \mathbb{Z} / 2 \mathbb{Z}$ has 
order 2 or $\infty$ by Lemma 3.6, it follows that $\alpha=2$. Since $h_{n}=x^{2}$ and $\phi\left(h_{n}\right)=\bar{a}^{2 n} \bar{b}^{2 n}$, it follows that $\phi(x)=\bar{a}^{n} \bar{b}^{n}$, and hence $\phi^{\prime}\left(x^{\prime}\right)=1$ or $\bar{a}^{\prime} \bar{b}^{\prime}$ by the same argument as in Case (b). However, since $h_{n}=x^{2}$, it follows that $x^{\prime 2}=1$, and hence $\phi^{\prime}\left(x^{\prime}\right)=\bar{a}^{\prime}$ or $\bar{b}^{\prime}$ by the same argument as in Case (b). This is a contradiction. Thus $G_{n}$ is not a classical link group.

Now we will show that $S_{n}$ and $S_{m}$ are not equivalent for $n \neq m$. The center $Z_{1}$ of $G_{1}$ is a free abelian group of rank 2 (see the proof of Lemma 3.4), while, for $n>1$, the center $Z_{n}$ of $G_{n}$ is an infinite cyclic group by Lemma 3.5; thus it suffices to show in the case when $n, m>1$. The abelianization of $G_{n} / Z_{n}$ for $n>1$ is $\mathbb{Z} \times \mathbb{Z} / 2 n \mathbb{Z}$; thus $G_{n}$ is not isomorphic to $G_{m}$ for $n \neq m$, and hence $S_{n} \not S_{m}$ for $n \neq m$.

A 2-link is a surface link whose components are homeomorphic to 2-spheres. It is known (see Hillman [17, Chapter 3, Corollary 2]) that if the center of a $\mu$-component $2-$ link group with $\mu>1$ is nontrivial, then the center must be a torsion group. Hence we have a corollary.

Corollary 3.3 The link group of the 2-component torus-covering $T^{2}$-link of Theorem 3.2 is not a 2-component 2-link group.

Proof For any $n>0$, the center $Z_{n}$ of $G_{n}$ is nontrivial and torsion free by Lemma 3.4 and Lemma 3.5; thus the conclusion follows from [17, Chapter 3, Corollary 2].

Lemma 3.4 The group $G_{1}$ of Theorem 3.2 is not a classical link group.

Proof Let $Z_{1}$ be the subgroup of $G_{1}$ generated by $\left\{a^{2}, b^{2}\right\}$. We will show that $Z_{1}$ is the center of $G_{1}$, as follows. Let $N$ be a normal subgroup of $G_{1}$. If the center of $G_{1} / N$ is trivial, then $N$ contains the center of $G_{1}$. Since $Z_{1}$ consists of central elements, $Z_{1}$ is a normal subgroup of $G_{1}$ such that $Z_{1}$ is contained in the center of $G_{1}$. Hence it suffices to show that the center of the quotient group $G_{1} / Z_{1}$ is trivial. Since $G_{1} / Z_{1}=\mathbb{Z} / 2 \mathbb{Z} * \mathbb{Z} / 2 \mathbb{Z}$, the center of $G_{1} / Z_{1}$ is trivial; thus $Z_{1}$ is the center of $G_{1}$. Let us take the abelianization map $\phi$ given by (3-4). Since $\phi\left(a^{2}\right)=\bar{a}^{2}$ and $\phi\left(b^{2}\right)=\bar{b}^{2}$, the center $Z_{1}$ is a free abelian group of rank 2 ; thus $Z_{1}$ is generated by two generators. Hence it follows from Burde and Murasugi [9] that if $G_{1}$ is a classical link group, then it is isomorphic to $\mathbb{Z} \times \mathbb{Z}$ (type (a)): $G_{1}$ is commutative. However, since the image of $G_{1}$ by the natural epimorphism $f$ given by (3-3) is a noncommutative group $\mathbb{Z} / 2 \mathbb{Z} * \mathbb{Z} / 2 \mathbb{Z}, G_{1}$ is not commutative. This is a contradiction. Thus $G_{1}$ is not a classical link group. 
Lemma 3.5 Let us consider the group $G_{n}$ of Theorem 3.2. For $n>1$, let $Z_{n}$ be the subgroup of $G_{n}$ generated by $h_{n}=\left(a^{2} b^{2}\right)^{n}$. Then $Z_{n}$ is the center of $G_{n}$. Moreover $Z_{n}$ is an infinite cyclic group.

Proof By the same argument as in the proof of Lemma 3.4, in order to show that $Z_{n}$ is the center of $G_{n}$, it suffices to show that the center of the quotient group $G_{n} / Z_{n}$ is trivial. We see that $G_{n} / Z_{n}=\left\langle a, b \mid\left(a^{2} b^{2}\right)^{n}=1\right\rangle$, which is an amalgamated product $\langle a\rangle *_{U}\left\langle b, x \mid x^{n}=1\right\rangle$, where $U=\left\langle a^{2}\right\rangle=\left\langle x b^{-2}\right\rangle=\mathbb{Z}$ and the amalgamation is given by $a^{2}=x b^{-2}$. Put $H_{1}=\langle a\rangle$ and $H_{2}=\left\langle b, x \mid x^{n}=1\right\rangle$. We can take $\{1, a\}$ as a set of right-handed coset representatives of $U$ in $H_{1}$.

Let $h$ be a central element of $G_{n} / Z_{n}=H_{1} * U H_{2}$. By Neumann [29] or Bogopolski [6, Theorem 11.3, page 73], $h$ is uniquely written as $h=u a^{\delta} c_{1} a c_{2} \cdots a c_{t} a^{\epsilon}$, where $u \in U$ and $c_{1}, \ldots, c_{t}$ are nontrivial elements of a set of right-handed coset representatives of $U$ in $H_{2}$ and $\delta, \epsilon \in\{0,1\}$, which is called a normal form. Since $a h=h a$, it follows that $a u a^{\delta} c_{1} a c_{2} \cdots a c_{t} a^{\epsilon}=u a^{\delta} c_{1} a c_{2} \cdots a c_{t} a^{\epsilon} a$. Since $u a=a u$ in the amalgamated product $H_{1} *_{U} H_{2}$, it follows that $u a^{\delta} a c_{1} a c_{2} \cdots a c_{t} a^{\epsilon}=u a^{\delta} c_{1} a c_{2} \cdots a c_{t} a a^{\epsilon}$, and hence $a c_{1} a c_{2} \cdots a c_{t}=c_{1} a c_{2} \cdots a c_{t} a$ as elements in $H_{1} * U H_{2}$.

If $t>0$, then $a c_{1} a c_{2} \cdots a c_{t}$ and $c_{1} a c_{2} \cdots a c_{t} a$ are in distinct normal forms, which is a contradiction. Hence $t=0$ and $h=u a^{\delta}=a^{k}$ for an integer $k$. Since $h b=b h$, $a^{k} b=b a^{k}$. If $k=1$, then $a b=b a$. In this case, if $b$ is not in $U$, then we can take $b$ as a nontrivial right-handed coset representative of $U$ in $H_{2}$. It follows that then $a b$ and $b a$ are in distinct normal forms, which is a contradiction. If $k=2 l+1$ (respectively $k=2 l$ ) for a nonzero integer $l$, then $a^{k} b=u a b$ and $b a^{k}=c a$ (respectively $a^{k} b=u b$ and $\left.b a^{k}=c\right)$, where in both cases $u=a^{2 l} \in U$ and $c=b\left(x b^{-2}\right)^{l}$. In these cases, if neither $b$ nor $c$ is in $U$ and we can take $b$ and $c$ as distinct right-handed coset representatives of $U$ in $H_{2}$, then $a^{k} b$ and $b a^{k}$ have distinct normal forms $u a b$ and $c a$ (respectively $u b$ and $c$ ), which is a contradiction. Then it follows that $k=0$ and hence $h=1$; thus the center of $G_{n} / Z_{n}$ is trivial.

It remains to show that neither $b$ nor $c=b\left(x b^{-2}\right)^{l}(l \neq 0)$ is in $U$ and we can take $b$ and $c$ as distinct right-handed coset representatives of $U$ in $H_{2}$. The group $H_{2}=\left\langle b, x \mid x^{n}=1\right\rangle$ is the free product of $\langle b\rangle$ and $\left\langle x \mid x^{n}=1\right\rangle$. By [29; 6, Theorem 11.3, page 73] again, every element of $H_{2}$ has a normal form $b_{1}^{\delta} x_{1} b_{2} x_{2} \cdots b_{t} x_{t}^{\epsilon}$, where $b_{1}, b_{2}, \ldots, b_{t}$ (respectively $x_{1}, x_{2}, \ldots, x_{t}$ ) are nontrivial elements of $\langle b\rangle$ (respectively $\left\langle x \mid x^{n}=1\right\rangle$ ) and $\delta, \epsilon \in\{0,1\}$. Let us determine the normal forms of $b$ and $c$. Put $l_{0}=|l|$, a positive integer. We can see that $b$ has a normal form $b$, and $c=b\left(x b^{-2}\right)^{l}$ has a normal form $b\left(x b^{-2}\right)^{l_{0}}$ (respectively $\left.b^{3} x^{-1}\left(b^{2} x^{-1}\right)^{l_{0}-1}\right)$ if $l>0$ (respectively $l<0$ ). Further, an element of $U=\left\langle x b^{-2}\right\rangle$ in $H_{2}$ has a normal form $1,\left(x b^{-2}\right)^{m_{0}}$ or $\left(b^{2} x^{-1}\right)^{m_{0}}$, where $m_{0}$ is a positive integer. Hence, by the 
uniqueness of normal forms, we can see that neither $b$ nor $c$ is in $U$. Similarly, an element of $U b$ has a normal form $b,\left(x b^{-2}\right)^{m_{0}-1} x b^{-1}$ or $\left(b^{2} x^{-1}\right)^{m_{0}} b$, and an element of $U c=U b\left(x b^{-2}\right)^{l}$ has a normal form $b\left(x b^{-2}\right)^{l_{0}}, b^{3} x^{-1}\left(b^{2} x^{-1}\right)^{l_{0}-1}$, $\left(x b^{-2}\right)^{m_{0}-1} x b^{-1}\left(x b^{-2}\right)^{l_{0}},\left(x b^{-2}\right)^{m_{0}-1} x b x^{-1}\left(b^{2} x^{-1}\right)^{l_{0}-1},\left(b^{2} x^{-1}\right)^{m_{0}} b\left(x b^{-2}\right)^{l_{0}}$ or $\left(b^{2} x^{-1}\right)^{m_{0}} b^{3} x^{-1}\left(b^{2} x^{-1}\right)^{l_{0}-1}$. By the uniqueness of normal forms, we can see that $U b \neq U c$. Thus neither $b$ nor $c$ is in $U$ and we can take $b$ and $c$ as distinct right-handed coset representatives of $U$ in $H_{2}$, and it follows that the center of $G_{n} / Z_{n}$ is trivial. Therefore $Z_{n}$ is the center of $G_{n}$.

Let us take the abelianization map $\phi$ given by (3-4). Since the image $\phi\left(h_{n}\right)$ is $\bar{a}^{2 n} \bar{b}^{2 n}$, the center $Z_{n}(n>1)$ is an infinite cyclic group.

Lemma 3.6 Let us consider the group $\mathbb{Z} / p \mathbb{Z} * \mathbb{Z} / q \mathbb{Z}$, the free product of $\mathbb{Z} / p \mathbb{Z}$ and $\mathbb{Z} / q \mathbb{Z}$, where $p, q$ are positive integers greater than one. Then the order of a nontrivial element $\mathbb{Z} / p \mathbb{Z} * \mathbb{Z} / q \mathbb{Z}$ is either $\infty$, a divisor of $p$ or a divisor of $q$. Further, if the order is finite, then the element can be written as a conjugate of an element of the same order in $\mathbb{Z} / p \mathbb{Z}$ or $\mathbb{Z} / q \mathbb{Z}$.

Proof Let $z$ be a nontrivial element of $\mathbb{Z} / p \mathbb{Z} * \mathbb{Z} / q \mathbb{Z}$. By Neumann [29] or Bogopolski [6, Theorem 11.3, page 73], $z$ has a normal form

$$
\begin{aligned}
& x_{1} y_{1} x_{2} y_{2} \cdots x_{t} y_{t} \\
& x_{1} y_{1} x_{2} y_{2} \cdots x_{t-1} y_{t-1} x_{t} \\
& y_{1} x_{2} y_{2} \cdots x_{t} y_{t} \\
& y_{1} x_{2} y_{2} \cdots x_{t-1} y_{t-1} x_{t}
\end{aligned}
$$

where $t$ is an integer with $t>1$ in (3-8) and $t>0$ otherwise, and $x_{1}, x_{2}, \ldots, x_{t}$ (respectively $y_{1}, y_{2}, \ldots, y_{t}$ ) are nontrivial elements of $\mathbb{Z} / p \mathbb{Z}$ (respectively $\mathbb{Z} / q \mathbb{Z}$ ).

In cases (3-5) and (3-8), $z^{l}$ has a normal form which is not 1 for any positive integer $l$. Thus the order of $z$ is infinite.

In cases (3-6) and (3-7), we show the lemma using induction for $t$, as follows. If $t=1$, then $z=x_{1}$ (respectively $y_{1}$ ) for (3-6) (respectively (3-7)) and the order of $z$ is a divisor of $p$ (respectively $q$ ). Now let us assume that if $t<s$, then the order of any element $z$ with the normal form (3-6) or (3-7) is infinite or a divisor of $p$ or $q$. Let us consider $z$ with the normal form (3-6) with $t=s$. If $x_{1} x_{t}=1$, then $x_{1}^{-1} z x_{1}$ has a normal form (3-7) with $t=s-1$; thus, by the assumption, the statement of the lemma holds. If $x_{1} x_{t} \neq 1$, then $z^{l}$ has a nontrivial normal form for any positive integer $l$; thus the order of $z$ is infinite. For $z$ with the normal form (3-7), we can apply the same argument. Further we can see that if the order $\operatorname{ord}(z)$ of $z$ is finite, ie a divisor of $p$ 
or $q$, then $z$ can be written as $\xi^{-1} x \xi$, where $\xi \in \mathbb{Z} / p \mathbb{Z} * \mathbb{Z} / q \mathbb{Z}$, and $x$ is an element of order $\operatorname{ord}(z)$ in $\mathbb{Z} / p \mathbb{Z}$ or $\mathbb{Z} / q \mathbb{Z}$.

We can consider the knot version of Theorem 3.2.

Theorem 3.7 Put $S_{n}=\mathcal{S}_{4}\left(\sigma_{1} \sigma_{3}, \Delta^{2 n+1}\right)$, where $n$ is a positive integer. Then the knot group of $S_{n}$ is not a classical knot group. Moreover, $S_{n}$ and $S_{m}$ are not equivalent for $n \neq m$.

The torus-covering $T^{2}$-knot $S_{0}=\mathcal{S}_{4}\left(\sigma_{1} \sigma_{3}, \Delta\right)$ is unknotted (see Corollary 4.4).

Proof By Proposition 3.1, the knot group $G_{n}$ of $S_{n}$ is computed as follows. Let $x_{1}, \ldots, x_{4}$ be the generators. Then the relations concerning the basis braid $\sigma_{1} \sigma_{3}$ are $x_{1}=x_{2}$ and $x_{3}=x_{4}$. The other relations concerning the basis braid $\Delta^{2 n+1}$ are:

$$
\begin{aligned}
& x_{1}=\left(x_{1} x_{2} x_{3} x_{4}\right)^{n} x_{1} x_{2} x_{3} x_{4} x_{3}^{-1} x_{2}^{-1} x_{1}^{-1}\left(x_{1} x_{2} x_{3} x_{4}\right)^{-n} \\
& x_{2}=\left(x_{1} x_{2} x_{3} x_{4}\right)^{n} x_{1} x_{2} x_{3} x_{2}^{-1} x_{1}^{-1}\left(x_{1} x_{2} x_{3} x_{4}\right)^{-n} \\
& x_{3}=\left(x_{1} x_{2} x_{3} x_{4}\right)^{n} x_{1} x_{2} x_{1}^{-1}\left(x_{1} x_{2} x_{3} x_{4}\right)^{-n} \\
& x_{4}=\left(x_{1} x_{2} x_{3} x_{4}\right)^{n} x_{1}\left(x_{1} x_{2} x_{3} x_{4}\right)^{-n}
\end{aligned}
$$

Putting $a=x_{1}=x_{2}$ and $b=x_{3}=x_{4}$, we have:

$$
G_{n}=\left\langle a, b \mid b\left(a^{2} b^{2}\right)^{n}=\left(a^{2} b^{2}\right)^{n} a, a\left(a^{2} b^{2}\right)^{n+1}=\left(a^{2} b^{2}\right)^{n+1} b\right\rangle
$$

Let us assume that $G_{n}$ is a classical knot group. Let $Z_{n}$ be the subgroup of $G_{n}$ generated by $h_{n}=\left(a^{2} b^{2}\right)^{2 n+1}$, which is a central element. By Lemma 3.8, $Z_{n}$ is the center of $G_{n}$. Further, $Z_{n}$ is an infinite cyclic group. It is known (see Burde and Zieschang [10]) that if the center of a classical knot group is nontrivial, then the knot is a torus knot. Hence, by the assumption, $G_{n}$ is isomorphic to a torus knot group. Let $G_{p, q}$ be the $(p, q)$-torus knot group isomorphic to $G_{n}$, where $p$ and $q$ are coprime positive integers. Let $Z_{p, q}$ be the center of $G_{p, q}$. Then $G_{p, q}=\langle x, y|$ $\left.x^{p}=y^{q}\right\rangle$ and $Z_{p, q}$ is generated by $h=x^{p}=y^{q}$. Put $G_{p, q}^{\prime}=G_{p, q} / Z_{p, q}$, which is $\left\langle x, y \mid x^{p}=y^{q}=1\right\rangle=\mathbb{Z} / p \mathbb{Z} * \mathbb{Z} / q \mathbb{Z}$. The abelianization of $G_{p, q}^{\prime}$ is isomorphic to $\mathbb{Z} / p \mathbb{Z} \times \mathbb{Z} / q \mathbb{Z}$.

Consider the quotient group $G_{n}^{\prime}=G_{n} / Z_{n}$. By (3-9) in the proof of Lemma 3.8, $G_{n}^{\prime}=\left\langle a, x \mid x^{2 n+1}=\left(a^{2} x^{n}\right)^{2}=1\right\rangle$. The abelianization of $G_{n}^{\prime}$ is presented by $\left\langle a, x \mid x^{2 n+1}=\left(a^{2} x^{n}\right)^{2}=1, a x=x a\right\rangle$, which equals $\left\langle a \mid a^{4(2 n+1)}\right\rangle=\mathbb{Z} / 4(2 n+1) \mathbb{Z}$. Since $G_{p, q}$ and $G_{n}$ are isomorphic, so are the abelianizations of $G_{p, q}^{\prime}$ and $G_{n}^{\prime}$. Hence, comparing the order of the groups we see that $p q=4(2 n+1)$. Since $G_{n}^{\prime}$ has an element of order $2 n+1$ by Lemma 3.9, and the order of a nontrivial torsion element 
of $G_{p, q}^{\prime}$ is a divisor of $p$ or $q$ by Lemma 3.6, it follows that $2 n+1$ is a divisor of $p$ or $q$. Hence we can determine coprime positive integers $p$ and $q$ by $p=4$ and $q=2 n+1$.

For any element $z$ of order 2 in $G_{4,2 n+1}^{\prime}=\mathbb{Z} / 4 \mathbb{Z} * \mathbb{Z} /(2 n+1) \mathbb{Z}, z$ can be written as $z=z^{\prime 2}$ for some element $z^{\prime}$ of order 4 by Lemma 3.10. Since $y=a^{2} x^{n} \in G_{n}^{\prime}$ is of order 2 by Lemma 3.9, and $G_{n}^{\prime}$ and $G_{4,2 n+1}^{\prime}$ are isomorphic, there is an element $y^{\prime} \in G_{n}^{\prime}$ with $y=y^{\prime 2}$, and hence $G_{n}^{\prime}=\left\langle a, x, y^{\prime} \mid x^{2 n+1}=1, y^{\prime 4}=1, a^{2} x^{n}=y^{\prime 2}\right\rangle$. Let $N_{w}$ be the normal subgroup of $G_{4,2 n+1}^{\prime}$ generated by an element $w$ of order $2 n+1$. The quotient group $G_{4,2 n+1}^{\prime} / N_{w}$ does not depend on the choice of $w$ and $G_{4,2 n+1}^{\prime} / N_{w}=\mathbb{Z} / 4 \mathbb{Z}$ by Lemma 3.11. We will denote it by $G_{4,2 n+1}^{\prime} / N$. Let $N_{x}$ be the normal subgroup of $G_{n}^{\prime}$ generated by $x$. Since $x$ has order $2 n+1$ by Lemma 3.9, $G_{n}^{\prime} / N_{x}$ is isomorphic to $G_{4,2 n+1}^{\prime} / N=\mathbb{Z} / 4 \mathbb{Z}: G_{n}^{\prime} / N_{x}$ is abelian. Adding the relation $x=1$ to the presentation of $G_{n}^{\prime}$, we see that $G_{n}^{\prime} / N_{x}=\left\langle a, y^{\prime} \mid y^{\prime 4}=1, a^{2}=y^{\prime 2}\right\rangle$. Since there is a natural epimorphism $f$ from $G_{n}^{\prime} / N_{x}$ onto $\mathbb{Z} / 2 \mathbb{Z} * \mathbb{Z} / 2 \mathbb{Z}$ with the basis $f(a)$ and $f\left(y^{\prime}\right), G_{n}^{\prime} / N_{x}$ is not abelian. This is a contradiction. Thus $G_{n}$ is not a classical knot group. Since the abelianization of $G_{n}^{\prime}$ is $\mathbb{Z} / 4(2 n+1) \mathbb{Z}$, it follows that $S_{n} \nsim S_{m}$ for $n \neq m$.

Lemma 3.8 Let us consider the group $G_{n}$ of Theorem 3.7. For $n>0$, let $Z_{n}$ be the subgroup of $G_{n}$ generated by $h_{n}=\left(a^{2} b^{2}\right)^{2 n+1}$. Then $Z_{n}$ is the center of $G_{n}$. Moreover $Z_{n}$ is an infinite cyclic group.

Proof Since $h_{n}$ is a central element, by the same argument as in the proof of Lemma 3.4, in order to show that $Z_{n}$ is the center of $G_{n}$, it suffices to show that the center of $G_{n}^{\prime}=G_{n} / Z_{n}$ is trivial. The quotient group $G_{n}^{\prime}=G_{n} / Z_{n}$ is presented by $\left\langle a, b, x \mid x=a^{2} b^{2}, b x^{n}=x^{n} a, a x^{n+1}=x^{n+1} b, x^{2 n+1}=1\right\rangle$. By eliminating $b$ by $b=x^{n} a x^{-n}$, we have

$$
G_{n}^{\prime}=\left\langle a, x \mid x^{2 n+1}=\left(a^{2} x^{n}\right)^{2}=1\right\rangle
$$

which is an amalgamated product $\langle a\rangle *_{U}\left\langle x, y \mid x^{2 n+1}=1, y^{2}=1\right\rangle$, where $U=\left\langle a^{2}\right\rangle=$ $\left\langle y x^{-n}\right\rangle=\mathbb{Z}$ and the amalgamation is given by $a^{2}=y x^{-n}$. We can show that the center of $G_{n}^{\prime}$ is trivial by the following argument similar to the proof of Lemma 3.5, as follows. Put $H_{1}=\langle a\rangle$ and $H_{2}=\left\langle x, y \mid x^{2 n+1}=1, y^{2}=1\right\rangle$. Note that $H_{2}$ is a free product of $\left\langle x \mid x^{2 n+1}=1\right\rangle=\mathbb{Z} /(2 n+1) \mathbb{Z}$ and $\left\langle y \mid y^{2}=1\right\rangle=\mathbb{Z} / 2 \mathbb{Z}$. By Neumann [29] or Bogopolski [6, page73, Theorem 11.3], any element of $G_{n}^{\prime}=H_{1} *_{U} H_{2}$ has a normal form $u a^{\delta} c_{1} a c_{2} \cdots a c_{t} a^{\epsilon}$, where $u \in U$ and $c_{1}, \ldots, c_{t}$ are nontrivial elements of a set of right handed coset representatives of $U$ in $H_{2}$ and $\delta, \epsilon \in\{0,1\}$.

Let $h$ be a central element of $G_{n}^{\prime}$. By the same argument as in the proof of Lemma 3.5, by using normal forms, we see that $h=a^{k}$ for an integer $k$. Since $h x=x h, a^{k} x=x a^{k}$. 
If $k=2 l+1$ (respectively $2 l$ ) for a nonzero integer $l$, then $a^{k} x=u a x$ and $x a^{k}=c a$ (respectively $a^{k} x=u x$ and $x a^{k}=c$ ), where $u=a^{2 l} \in U$ and $c=x\left(y x^{-n}\right)^{l}$ in both cases. If neither $x$ nor $c$ is in $U$ and we can choose $x$ and $c$ as distinct right-handed coset representatives of $U$ in $H_{2}$, then in both cases $a^{k} x$ and $x a^{k}$ have distinct normal forms, which is a contradiction. Then $k=0$, and it follows that $h=1$.

It remains to show that neither $x$ nor $c=x\left(y x^{-n}\right)^{l}(l \neq 0)$ is in $U$ and we can choose $x$ and $c$ as distinct right-handed coset representatives of $U$ in $H_{2}$. By [29;6, Theorem 11.3, page 73=, any element of the free product $H_{2}=\left\langle x \mid x^{2 n+1}=1\right\rangle *\langle y|$ $\left.y^{2}=1\right\rangle$ has a normal form $x_{1}^{\delta} y x_{2} y \cdots x_{t} y^{\epsilon}$, where $x_{1}, x_{2}, \ldots, x_{t}$ are nontrivial elements of $\left\langle x \mid x^{2 n+1}=1\right\rangle$ and $\delta, \epsilon \in\{0,1\}$. Let us determine the normal forms of $x$ and $c$. Put $l_{0}=|l|$, a positive integer. We can see that $x$ has a normal form $x$, and $c=x\left(y x^{-n}\right)^{l}$ has a normal form $x\left(y x^{-n}\right)^{l_{0}}$ (respectively $\left.x^{n+1} y\left(x^{n} y\right)^{l_{0}-1}\right)$ if $l>0$ (respectively $l<0$ ). Further, an element of $U=\left\langle y x^{-n}\right\rangle$ has a normal form either $1,\left(y x^{-n}\right)^{m_{0}}$ or $\left(x^{n} y\right)^{m_{0}}$, where $m_{0}$ is a positive integer. Hence, by the uniqueness of normal forms, neither $x$ nor $c$ is in $U$. Similarly, if $n=1$ (respectively $n>1)$, then an element of $U x$ has a normal form either $x,\left(y x^{-1}\right)^{m_{0}-1} y$ or $(x y)^{m_{0}} x$ (respectively $x,\left(y x^{-n}\right)^{m_{0}-1} y x^{-n+1}$ or $\left.\left(x^{n} y\right)^{m_{0}} x\right)$. Hence in both cases $c$ is not an element of $U x$. Thus neither $x$ nor $c$ is in $U$ and we can choose $x$ and $c$ as distinct right-handed coset representatives of $U$ in $H_{2}$, and it follows that the center of $G_{n}^{\prime}$ is trivial. Thus $Z_{n}$ is the center. Considering the abelianization map of $G_{n}$, we see that $Z_{n}$ is an infinite cyclic group.

Lemma 3.9 The element $x$ (respectively $y=a^{2} x^{n}$ ) of $G_{n}^{\prime}$ of Theorem 3.7 (see (3-9)) is of order $2 n+1$ (respectively 2 ).

Proof By Lemma 3.8, $G_{n}^{\prime}$ is an amalgamated product. Seeing the normal forms of the powers of $x$ and $y$, we can show that the order of $x$ is $2 n+1$ and the order of $y$ is 2 .

Lemma 3.10 For any element $z$ of order 2 in $\mathbb{Z} / 4 \mathbb{Z} * \mathbb{Z} /(2 n+1) \mathbb{Z}, z$ can be written as $z=z^{\prime 2}$ for some element $z^{\prime}$ of order 4 .

Proof The order of $z$ is 2 . It is a divisor of 4 , and it is not a divisor of $2 n+1$. Thus it follows from Lemma 3.6 that $z=\xi^{-1} u^{2} \xi$, where $\xi \in \mathbb{Z} / 4 \mathbb{Z} * \mathbb{Z} /(2 n+1) \mathbb{Z}$ and $u$ is a generator of $\mathbb{Z} / 4 \mathbb{Z}$. Thus $z$ can be written as $z=z^{\prime 2}$, where $z^{\prime}=\xi^{-1} u \xi$. Since the order of $u$ is 4 , so is the order of $z^{\prime}$.

Lemma 3.11 Put $G_{4,2 n+1}^{\prime}=\mathbb{Z} / 4 \mathbb{Z} * \mathbb{Z} /(2 n+1) \mathbb{Z}$, and let $N_{w}$ be a normal subgroup of $G_{4,2 n+1}^{\prime}$ generated by an element $w$ of order $2 n+1$. Then $G_{4,2 n+1}^{\prime} / N_{w}$ does not depend on the choice of $w$, and $G_{4,2 n+1}^{\prime} / N_{w}=\mathbb{Z} / 4 \mathbb{Z}$. 
Proof Since $w$ has order $2 n+1, w=\xi^{-1} v^{k} \xi$ by Lemma 3.6, where $\xi \in \mathbb{Z} / 4 \mathbb{Z} *$ $\mathbb{Z} /(2 n+1) \mathbb{Z}$ and $v$ is a generator of $\mathbb{Z} /(2 n+1) \mathbb{Z}$, and $k$ is an integer such that $v^{k}$ has order $2 n+1$. Put $X=\{\overline{k l} \mid l=1,2, \ldots, 2 n\}$, where $\overline{k l}=k l \bmod 2 n+1$. If $\overline{k l}=\overline{k l^{\prime}}$ for $l \neq l^{\prime}\left(0<l, l^{\prime}<2 n+1\right)$, then $k\left|l-l^{\prime}\right| \equiv 0 \bmod 2 n+1$, and it follows that the order of $w$ is a divisor of $\left|l-l^{\prime}\right|<2 n$. Then the order is smaller than $2 n+1$, which is a contradiction. Hence, if $l \neq l^{\prime}\left(0<l, l^{\prime}<2 n+1\right)$, then $\overline{k l} \neq \overline{k l^{\prime}}$. Since $v^{k}$ has order $2 n+1, v^{k l} \neq 1$ for $0<l<2 n+1$; thus $\overline{0} \notin X$. Thus $X=\{\overline{1}, \overline{2}, \ldots, \overline{2 n}\}$, and hence $X$ contains $\overline{1}$. Hence $\overline{k l_{0}}=\overline{1}$ for some integer $l_{0}$, and it follows that $w^{l_{0}}=\xi^{-1} v^{k l_{0}} \xi=\xi^{-1} v \xi$. Since $v=\xi w^{l_{0}} \xi^{-1}$, we have $v \in N_{w}$. Since $N_{w}$ contains the generator $v$ of $N_{v}$, we have $N_{v} \subset N_{w}$. Similarly, since $w=\xi^{-1} v^{k} \xi$, we have $N_{w} \subset N_{v}$. Hence $N_{v}=N_{w}$. Thus $G_{4,2 n+1}^{\prime} / N_{w}=G_{4,2 n+1}^{\prime} / N_{v}=\mathbb{Z} / 4 \mathbb{Z}$.

Using the results of Theorem 3.2 and Theorem 3.7, we have the following theorem.

Theorem 3.12 For an integer $l>1, \mathcal{S}_{4}\left(\sigma_{1} \sigma_{3}, \Delta^{l}\right)$ is not equivalent to either a spun $T^{2}$-link, a turned spun $T^{2}$-link or the split union of spun $T^{2}$-links and turned spun $T^{2}$-links.

Proof Since the link group of the spun $T^{2}$-link or the turned spun $T^{2}$-link of a classical link $L$ is isomorphic to the link group of $L$ (see Livingston [26] and Boyle [8]), it is classical. Thus the link group of the split union of spun $T^{2}$-links and turned spun $T^{2}$-links is also classical. However, the link group of $\mathcal{S}_{4}\left(\sigma_{1} \sigma_{3}, \Delta^{l}\right)(l>1)$ is not classical by Theorem 3.2 and Theorem 3.7. Thus we have the conclusion.

\section{Ribbon torus-covering $T^{2}$-links}

In this section we show Theorem 4.1: for certain basis $m n$-braids, $\mathcal{S}_{m n}(a, b)$ is ribbon. As a corollary, we can see that the torus-covering $T^{2}$-link of Theorem 3.12 is ribbon (Corollary 4.2).

Let $M$ be a disjoint union of a finite number of handlebodies. The image of $M$ into $\mathbb{R}^{4}$ by an immersion $\phi$ is called a 3-ribbon (see Yanagawa [32]) if the singularity set consists of ribbon singularities, ie the self-intersection of $\phi(M)$ consists of a finite number of mutually disjoint 2-disks, and for each 2-disk $D$, the preimage $\phi^{-1}(D)$ consists of a pair of 2-disks $D^{\prime}, D^{\prime \prime}$ such that $D^{\prime} \cap D^{\prime \prime}=\varnothing, D^{\prime} \subset \operatorname{Int} M$ and $\partial D^{\prime \prime}=D^{\prime \prime} \cap \partial M$. An oriented surface link is ribbon if it bounds a 3-ribbon (see [32]).

For an $m$-braid $b$, we denote by $b^{(n)}$ the $n$-parallel of $b$, ie $b^{(n)}$ is the $m n$-braid obtained from $b$ by replacing each string of $b$ with its $n$ parallel copies; see Figure 7 . For $n$-braids $b_{1}, b_{2}, \ldots, b_{m}$, we denote by $b_{1} \circ b_{2} \circ \ldots \circ b_{m}$ the $m n$-braid depicted in Figure 8. 


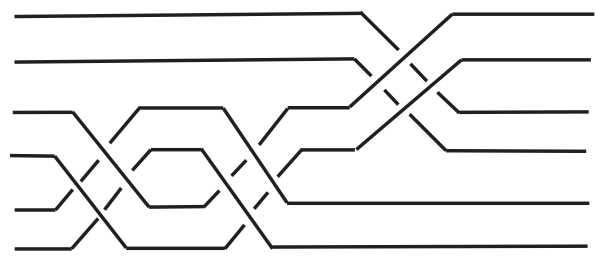

Figure 7: The 2-parallel $\left(\sigma_{1}^{2} \sigma_{2}^{-1}\right)^{(2)}$ of the 3-braid $\sigma_{1}^{2} \sigma_{2}^{-1}$

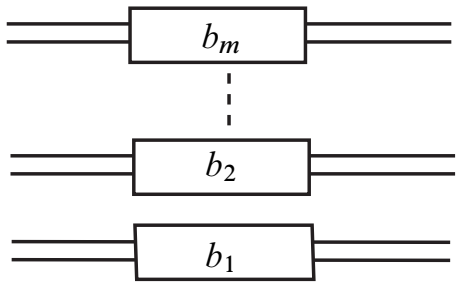

Figure 8: The $m n$-braid $b_{1} \circ b_{2} \circ \cdots \circ b_{m}$

Theorem 4.1 Let $\alpha$ be a classical $n$-braid whose closure $\hat{\alpha}$ is a trivial knot. Let $a$ and $b$ be $m n$-braids given by

$$
\begin{aligned}
& a=\overbrace{\alpha \circ \alpha \circ \cdots \circ \alpha}^{m} \\
& b=b^{\prime(n)} \cdot\left(\alpha^{l_{1}} \circ \alpha^{l_{2}} \circ \cdots \circ \alpha^{l_{m}}\right)
\end{aligned}
$$

where $b^{\prime}$ is an $m$-braid and $l_{j}$ is an integer $(j=1,2, \ldots, m)$; note that $a$ and $b$ are commutative. Then $\mathcal{S}_{m n}(a, b)$ is ribbon.

Proof Let the braid word presentation of $b^{\prime}$ be $b^{\prime}=\sigma_{i_{1}}^{\epsilon_{1}} \cdot \sigma_{i_{2}}^{\epsilon_{2}} \cdots \sigma_{i_{v}}^{\epsilon_{v}}$, where $i_{k} \in$ $\{1,2, \ldots, m-1\}$ and $\epsilon_{k} \in\{+1,-1\}$ for $k=1,2, \ldots, v$. Let $\pi: \mathbb{R}_{+}^{3} \times S^{1} \rightarrow \mathbb{R}_{+}^{3}$ be the projection. Let us take a solid torus $N(\mathbf{m}) \subset \mathbb{R}_{+}^{3}$ and a disk $D\left(x_{0}\right) \subset N(\mathbf{m})$ as in the proof of Proposition 2.11. Let us take the closure $\widehat{a}$ of $a$ in $N(\mathbf{m})$ as shown in Figure 9, where we take the $m$ parallel copies of $\hat{\alpha}$ in such a position that the identified corresponding ends are in $D\left(x_{0}\right)$.

We consider a surface link $S$ determined by the motion picture $S_{t}=\pi\left(S \cap\left(\mathbb{R}_{+}^{3} \times\{t\}\right)\right)$ along $S^{1}$, which is the orbit of the isotopy from $\hat{a}$ to $\hat{a}$, given by the composition of the following (1) and (2).

(1) Concerning $b^{(n)}$, let us take the isotopy from $\hat{a}$ to $\hat{a}$ as follows. For each $\left(\sigma_{i_{k}}^{\epsilon_{k}}\right)^{(n)}$, we consider the isotopy shown in Figure 10 if $\epsilon_{k}=+1$, and its inverse if $\epsilon_{k}=-1$. Further, we consider the composition of them for all $k$. 


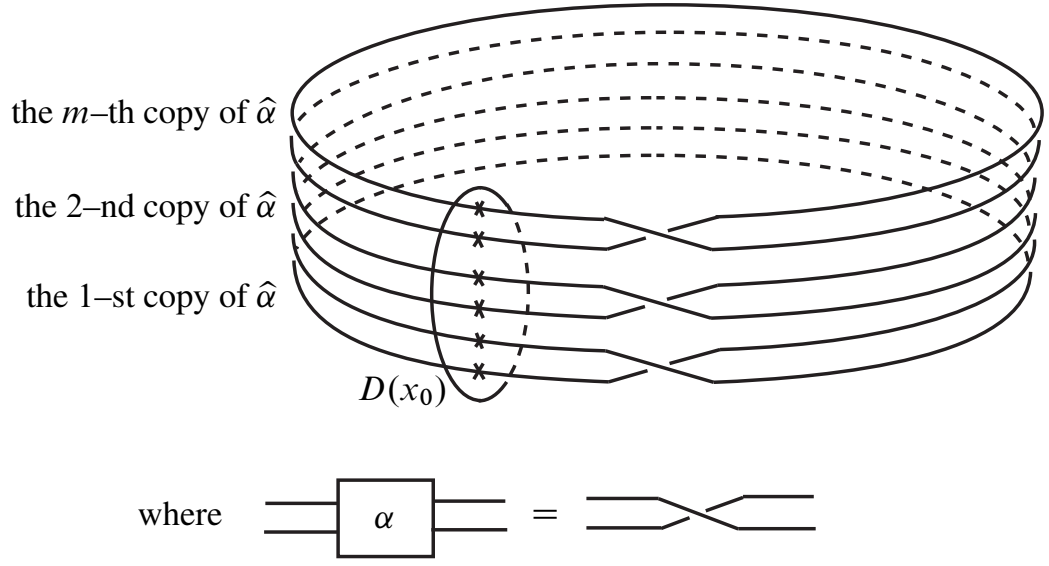

Figure 9: The closure $\hat{a}$ of $a$

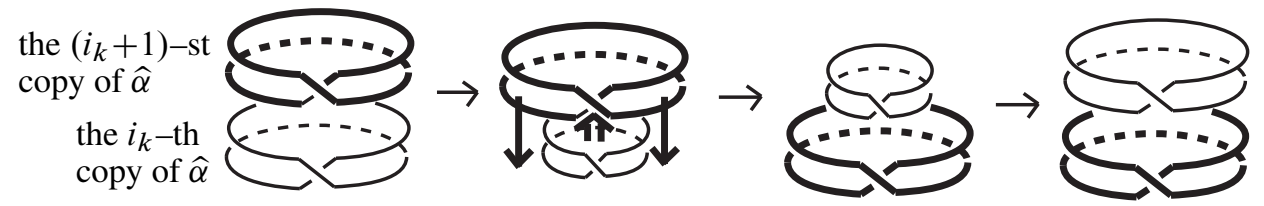

Figure 10: We consider this isotopy (1), concerning $\left(\sigma_{i_{k}}^{\epsilon_{k}}\right)^{(n)}$, if $\epsilon_{k}=+1$.

(2) Concerning $\alpha^{l_{1}} \circ \alpha^{l_{2}} \circ \cdots \circ \alpha^{l_{m}}$, let us take the isotopy from $\hat{a}$ to $\hat{a}$ which turns the $j$-th copy of $\hat{\alpha} l_{j}$ times as shown in Figure 11 , for each $j=1,2, \ldots, m$.

Since each isotopy is from $\widehat{a}$ to $\widehat{a},\left\{S_{t}\right\}$, and hence $S$, is well-defined.

the $j$-th copy of $\hat{\alpha}$
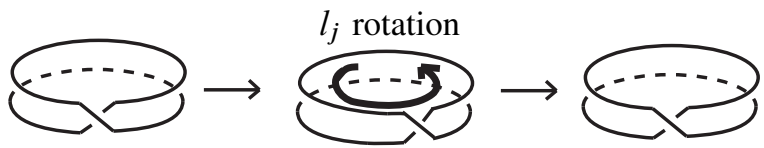

Figure 11: The isotopy (2), concerning $\alpha^{l_{1}} \circ \alpha^{l_{2}} \circ \cdots \circ \alpha^{l_{m}}$

Next we show that $S$ is equivalent to $\mathcal{S}_{m n}(a, b)$, as follows. It suffices to see that the orbit of $S_{t} \cap D\left(x_{0}\right)$ forms $b$. The orbit of $S_{t} \cap D\left(x_{0}\right)$ by the isotopy (1) is as in Figure 12; thus it forms $\left(\sigma_{i_{k}}^{\epsilon_{k}}\right)^{(n)}$. Since the isotopy (2) turns the $j$-th copy of $\hat{\alpha} l_{j}$ times $(j=1,2, \ldots, m)$, by the similar argument to the proof of Proposition 2.13, we can see that the orbit of $S_{t} \cap D\left(x_{0}\right)$ by this isotopy forms $\alpha^{l_{1}} \circ \alpha^{l_{2}} \circ \cdots \circ \alpha^{l_{m}}$. Thus, the orbit of $S_{t} \cap D\left(x_{0}\right)$ as a whole forms $\left(\sigma_{i_{1}}^{\epsilon_{1}} \sigma_{i_{2}}^{\epsilon_{2}} \cdots \sigma_{i_{v}}^{\epsilon_{v}}\right)^{(n)} \cdot\left(\alpha^{l_{1}} \circ \alpha^{l_{2}} \circ \cdots \circ \alpha^{l_{m}}\right)$, which is $b$. Thus $S$ is equivalent to $\mathcal{S}_{m n}(a, b)$ by Lemma 2.8 (2). 


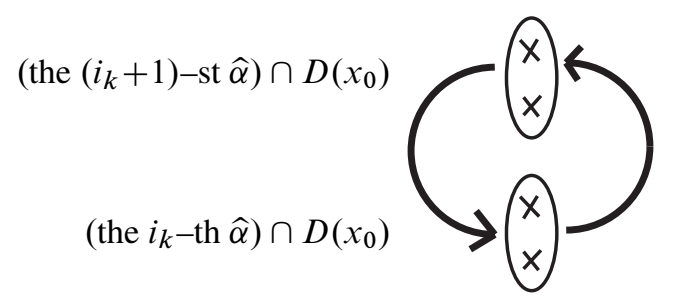

Figure 12: The orbit of $S_{t} \cap D\left(x_{0}\right)$ by the isotopy (1) concerning $\left(\sigma_{i_{k}}^{\epsilon_{k}}\right)^{(n)}$, if $\epsilon_{k}=+1$

Now let us construct an immersed 3-manifold $M$ such that $\partial M=S$, which is determined by $M_{t}=\pi\left(M \cap\left(\mathbb{R}_{+}^{3} \times\{t\}\right)\right)$ as follows. Since $\hat{\alpha}$ is a trivial knot, we can take a disk bounded by $\hat{\alpha}$ as shown in Figure 13. For each $S_{t}$, let $M_{t}$ be the union of such disks bounded by $S_{t}$. As the union of $M_{t}$, we naturally obtain an immersed 3-manifold $M$ such that $\partial M=S$.

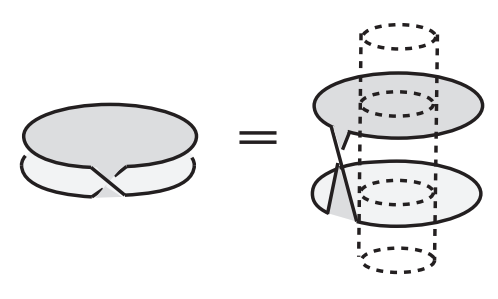

Figure 13: The disk bounded by $\hat{\alpha}$

In order to show that $\mathcal{S}_{m n}(a, b)$ is ribbon, it is sufficient to show that $M$ is a 3-ribbon, ie $M$ has only ribbon singularities. Since $M$ has no singularity in the motion picture of the isotopy (2), it is sufficient to show that the singularity in the motion picture of the isotopy (1) consists of ribbon singularities. Let us consider the singularity of $M$ in the motion picture of the isotopy (1) shown in Figure 10. This singularity is of the form of the singularity of the motion picture shown in Figure 14, and hence this singularity set is the disk itself. Therefore $M$ has only ribbon singularities.

Corollary 4.2 For any integer $l, \mathcal{S}_{4}\left(\sigma_{1} \sigma_{3}, \Delta^{l}\right)$ is ribbon.

Proof Put $a=\sigma_{1} \sigma_{3}$ and $b=\Delta^{l}$. Let $\alpha$ be a 2-braid $\sigma_{1}$. Then $\hat{\alpha}$ is a trivial knot, and $a=\alpha \circ \alpha$. By definition, $\sigma_{1}^{(2)}=\sigma_{2} \sigma_{1} \sigma_{3} \sigma_{2}$. Since $\Delta=\left(\sigma_{2} \sigma_{1} \sigma_{3} \sigma_{2}\right) \cdot \sigma_{1} \sigma_{3}=\sigma_{1}^{(2)} \cdot \sigma_{1} \sigma_{3}$, and $\sigma_{i} \cdot \sigma_{1}^{(2)}=\sigma_{1}^{(2)} \cdot \sigma_{j}$ for $\{i, j\}=\{1,3\}$, together with the fact that $\sigma_{1}$ and $\sigma_{3}$ commute, it follows that $\Delta^{l}$ can be written as $\Delta^{l}=\sigma_{1}^{l(2)} \cdot \sigma_{1}^{l} \sigma_{3}^{l}$; thus $b=b^{\prime(2)} \cdot\left(\alpha^{l} \circ \alpha^{l}\right)$, where $b^{\prime}=\sigma_{1}^{l}$. Thus the basis braids $a$ and $b$ have the required presentations of Theorem 4.1, and hence $\mathcal{S}_{4}\left(\sigma_{1} \sigma_{3}, \Delta^{l}\right)$ is ribbon by the theorem. 

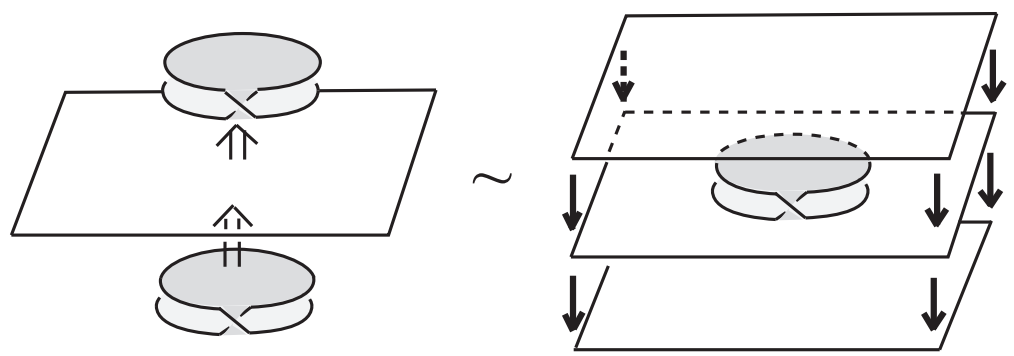

Figure 14: The motion picture of an upward move of the disk (the left picture) is equivalent to the motion picture of a downward move of a horizontal plane (the right picture)

So the torus-covering $T^{2}$-link of Theorem 3.12 is ribbon. Together with Theorem 3.12, this indicates the following corollary.

Corollary 4.3 For an integer $l>1, \mathcal{S}_{4}\left(\sigma_{1} \sigma_{3}, \Delta^{l}\right)$ has the following properties: (1) it can be presented by an $m$-chart on $S^{2}$ without white vertices, however (2) any $m$-chart on $T$ presenting it has at least one white vertex.

Proof Put $S=\mathcal{S}_{4}\left(\sigma_{1} \sigma_{3}, \Delta^{l}\right)$.

(1) Any ribbon surface link is presented by an $m$-chart on the standard 2-sphere $S^{2}$ without white vertices (see Kamada [20;24]). Since $S$ is ribbon by Corollary 4.2, it is presented by an $m$-chart on $S^{2}$ without white vertices.

(2) By Lemma 2.7 and Theorem 2.16, if an $m$-chart on $T$ presenting a torus-covering $T^{2}$-link does not have a white vertex, then it presents either a spun $T^{2}-$ link, a turned spun $T^{2}$-link or the split union of spun $T^{2}$-links and turned spun $T^{2}$-links. Since $S$ is not equivalent to such a surface link by Theorem 3.12, it cannot be presented by an $m$-chart on $T$ without white vertices.

Concerning $\mathcal{S}_{4}\left(\sigma_{1} \sigma_{3}, \Delta\right)$, we have the following corollary, by using the 3 -ribbon constructed in the proof of Theorem 4.1.

Corollary 4.4 The torus-covering $T^{2}-k n o t \mathcal{S}_{4}\left(\sigma_{1} \sigma_{3}, \Delta\right)$ is unknotted.

A 1-handle attaching to a surface link $S$ is a 3-ball $h$ embedded in $\mathbb{R}^{4}$ such that $S \cap h$ is a pair of 2-disks in $\partial h$. The closure (as a set) of $(S \cup \partial h)-(S \cap h)$ is a surface link. We call it the surface link obtained from $S$ by a 1 -handle surgery along a 1-handle $h$. A 2-handle attaching to $S$ is a 3-ball $h$ embedded in $\mathbb{R}^{4}$ such that $S \cap h$ is an annulus in $\partial h$. The closure (as a set) of $(S \cup \partial h)-(S \cap h)$ is a surface link. We call it the surface link obtained from $S$ by a 2-handle surgery along a 2 -handle $h$. The inverse operation of a 1 -handle surgery is a 2 -handle surgery, and vice versa. 
Proof By Corollary 4.2, $\mathcal{S}_{4}\left(\sigma_{1} \sigma_{3}, \Delta\right)$ is equivalent to $S=\partial M$ in the proof of Theorem 4.1. We use the notation of the theorem. By Corollary 4.2, $\alpha$ is the $2-$ braid $\sigma_{1}$, and the basis braids are the 4-braids given by $\alpha \circ \alpha$ and $\left(\sigma_{1}\right)^{(2)} \cdot(\alpha \circ \alpha)$. Since $\partial M_{0}=S_{0}$ is the closure of $\alpha \circ \alpha$, it consists of two components; thus the part of $M$ of the motion picture of the isotopy (1) consists of two connected components. Let us denote by $h$ one of the components containing the first copy of $\widehat{\alpha}$ in $\partial M_{0}$. Since $h$ is an embedded 3-ball such that $S \cap h$ is an annulus in $\partial h$ (see Figure 10), it is a 2-handle on $S$. Let $M^{\prime}=\operatorname{cl}(M-h)$, and put $S^{\prime}=\partial M^{\prime}$. Then $S^{\prime}$ is the surface link obtained from $S$ by a 2 -handle surgery along $h$. Since a 2 -handle surgery is the inverse operation of a 1 -handle surgery, $h$ is a 1 -handle on $S^{\prime}$, and $S$ is obtained from $S^{\prime}$ by a 1 -handle surgery along $h$. Since the singularity set of $M$ is contained in $h$ (see the proof of Theorem 4.1), $M^{\prime}$ is an embedded 3-ball with no singularity; thus $S^{\prime}$ is an unknotted sphere. It is known (see Boyle [7, Corollary 5]) that if a surface knot is unknotted, then the result of a 1-handle surgery for any 1-handle is also unknotted. Thus $S$, hence $\mathcal{S}_{4}\left(\sigma_{1} \sigma_{3}, \Delta\right)$, is unknotted.

\section{Quandle cocycle invariants}

It is known (see Asami and Satoh [2]) that the quandle cocycle invariant of a twistspun 2-knot of a classical knot $K$ can be presented by using the quandle cocycle invariants of a 1-tangle whose closure is $K$. In this section we present the quandle cocycle invariant of $\mathcal{S}_{m}\left(b, \Delta^{2 n}\right)$ for an $m$-braid $b$ (Theorem 5.2), by using the quandle cocycle invariants of the closure of $b$. Here $\Delta$ is a half-twist of a bundle of $m$ parallel strands. In Theorem 5.5, we calculate some concrete examples.

This section is organized as follows. In Section 5.1, we review the quandle cocycle invariants and the shadow cocycle invariants. Further, we give a certain 2-cocycle, which is determined from a 3 -cocycle. Using these terms, we give the statement of Theorem 5.2. In Section 5.2, we study triple points of $\mathcal{S}_{m}\left(b, \Delta^{2 n}\right)$, and prove Theorem 5.2. In Section 5.3, we show Theorem 5.5, using a dihedral quandle and Mochizuki's 3-cocycle.

\subsection{Quandle cocycle invariant of $\mathcal{S}_{m}\left(b, \Delta^{2 n}\right)$}

A quandle (see Joyce [19]) is a set $Q$ with a binary operation $*$ satisfying the following conditions:

(i) For any $x \in Q, x * x=x$.

(ii) For any $x, y \in Q$, there exists a unique $z \in Q$ such that $x=z * y$.

(iii) For any $x, y, z \in Q,(x * y) * z=(x * z) *(y * z)$. 
From now on, assume that $Q$ is a finite quandle, ie a quandle consisting of finitely many elements.

For an oriented classical link $L$ or an oriented surface link $S$, let us denote by $D$ a diagram of $L$ or $S$, ie the image of $L$ or $S$ by a generic projection to $\mathbb{R}^{2}$ or $\mathbb{R}^{3}$. In order to indicate crossing information of the diagram, we break the under-arc or the under-sheet into two pieces missing the over-arc or the over-sheet. Then the diagram is presented by a disjoint union of arcs, or compact surfaces which are called broken sheets. Let $B(D)$ be the set of such arcs or broken sheets. A $Q$-coloring for a diagram $D$ of $L$ or $S$ is a map $C: B(D) \rightarrow Q$ as in Figure 15. The image by $C$ is called the color.
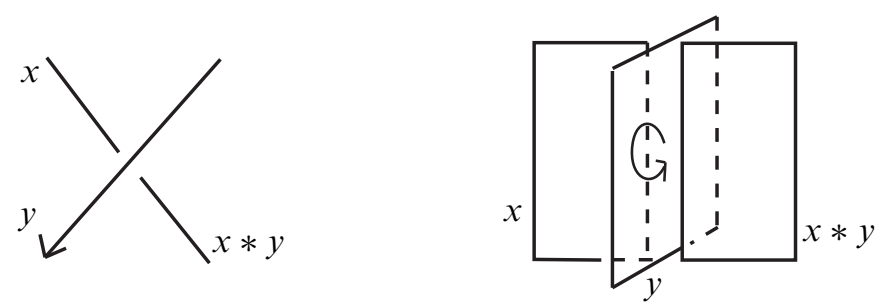

Figure 15: A $Q$-coloring $C$, where $x, y$ and $x * y$ are the colors of arcs or broken sheets given by $C$

Let $G$ be an abelian group. A 2 -cocycle with the coefficient group $G$ is a map $f: Q^{2} \rightarrow G$ satisfying

$$
f(s, u)+f(s * u, t * u)=f(s, t)+f(s * t, u)
$$

and

$$
f(s, s)=0
$$

for any $s, t, u \in Q$. A 3-cocycle is a map $f: Q^{3} \rightarrow G$ satisfying

$$
\begin{gathered}
f(s, t, u)+f(s * u, t * u, v)+f(s, u, v)=f(s * t, u, v) \\
+f(s, t, v)+f(s * v, t * v, u * v), \\
f(s, s, t)=0 \quad \text { and } \begin{array}{c}
f(s, t, t)=0
\end{array}
\end{gathered}
$$

for any $s, t, u, v \in Q$.

For a $Q$-coloring $C$ for a diagram $D$ of a classical link $L$ or a surface link $S$, we briefly review the quandle cocycle invariant as follows (for details see Carter, Jelsovsky, Kamada, Langford and Saito [11]), where $G$ is written multiplicatively. For the case 
of a classical link, at each crossing $r$ of the diagram $D$, the weight $W_{f}(r ; C)$ at $r$ for a 2-cocycle $f$ is given as in Figure 16. Put

$$
\Phi_{f}(L ; C)=\prod_{r \in X_{2}(D)} W_{f}(r ; C)
$$

where $X_{2}(D)$ is the set of the crossings of $D$. For the case of a surface link, at each triple point $t$ of the diagram $D$, the weight $W_{f}(t ; C)$ at $t$ for a 3 -cocycle $f$ is given as in Figure 17 (see [11, Sections 10 and 11] and also Carter and Saito [14, Proposition 4.43 (3)]). Put

$$
\Phi_{f}(S ; C)=\prod_{t \in X_{3}(D)} W_{f}(t ; C)
$$

where $X_{3}(D)$ is the set of the triple points of $D$. It is known [11] that $\Phi_{f}(L ; C)$ or

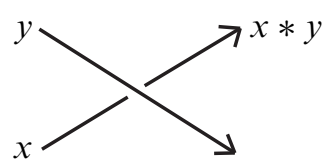

positive crossing weight $=f(x, y)$

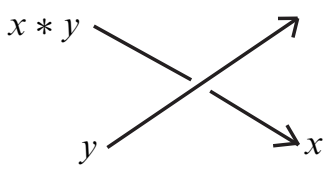

negative crossing weight $=f(x, y)^{-1}$

Figure 16: The weight at a crossing, where $x, y$ and $x * y$ are the colors of arcs by $C$, and $f$ is a $2-$ cocycle

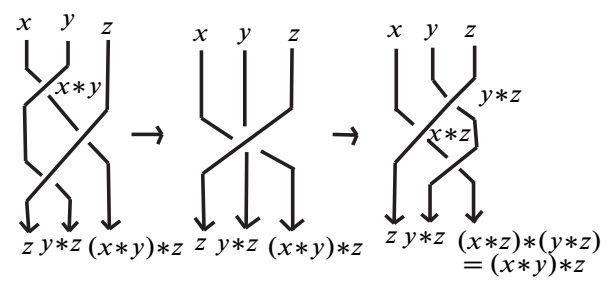

weight $=f(x, y, z)$
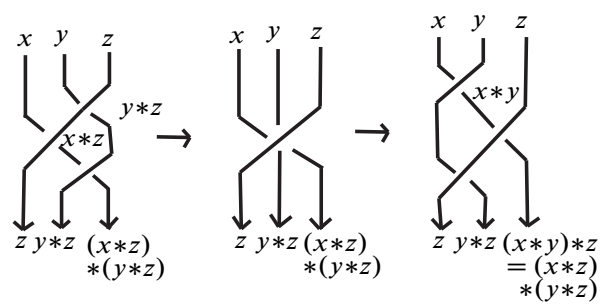

weight $=f(x, y, z)^{-1}$

Figure 17: The weight at a triple point, where the triple point is presented by a motion picture around it, and $x, y, z$, etc are the colors by $C$, and $f$ is a 3 -cocycle

$\Phi_{f}(S ; C)$ is an invariant of $L$ or $S$. We call it the quandle cocycle invariant of $L$ or $S$ associated with a $Q$-coloring $C$ (see [11]). Since $B(D)$ is a finite set, so is 
the set of $Q$-colorings for $D$. Let $\operatorname{Col}_{Q}(D)$ be the set of all the $Q$-colorings. Then define $\Phi_{f}(L)$ or $\Phi_{f}(S)$ by

$$
\Phi_{f}(X)=\sum_{C \in \operatorname{Col}_{Q}(D)} \Phi_{f}(X ; C) \in \mathbb{Z}[G]
$$

where $X=L$ or $S$, and $f$ is a 2-cocycle (respectively 3-cocycle) if $X=L$ (respectively $X=S$ ). We call $\Phi_{f}(X)$ the quandle cocycle invariant of $X$ associated with $f[11]$.

Next we define a shadow coloring for a classical link. For a diagram of a classical link with a given $Q$-coloring, its shadow color is determined from the color of the unbounded region (see Carter, Kamada and Saito [13]), which we will call the base color.

Let $C$ be a $Q$-coloring for a diagram $D$ of a classical link $L$. A shadow coloring of $D$ extending $C$ with the base color $x \in Q$ is a map $C_{x}^{*}: B^{*}(D) \rightarrow Q$, where $B^{*}(D)$ is the union of $B(D)$ and the set of regions of $\mathbb{R}^{2}$ separated by the immersed strings of the diagram $D$, satisfying the following conditions:

(i) $C_{x}^{*}$ restricted to $B(D)$ is coincident with $C$.

(ii) The color of the regions are as in Figure 18.

(iii) The color of the unbounded region is $x$.

By [13], $C_{x}^{*}$ exists uniquely for given $C$ and $x$. For a 3-cocycle $f$, let us define the weight at a positive (respectively negative) crossing $r$ by $W_{f}^{*}(r ; C, x)=f(w, y, z)$ (respectively $\left.f(w, y, z)^{-1}\right)$ in $G$, where $y, z$ and $w$ are the colors shown in Figure 18. Put:

$$
\Psi_{f}^{*}(L ; C, x)=\prod_{r \in X_{2}(D)} W_{f}^{*}(r ; C, x)
$$

It is known [13] that $\Psi_{f}^{*}(L ; C, x)$ is an invariant of $L$. We will call $\Psi_{f}^{*}(L ; C, x)$ the shadow cocycle invariant of $L$ associated with the $Q$-coloring $C$ and the base color $x$ (see [13]).

Let $\mathcal{R}_{y}: Q \rightarrow Q$ be a map defined by $\mathcal{R}_{y}(x)=x * y$ for $x, y \in Q$. Further, let $\mathcal{R}_{\varnothing}=$ $\operatorname{id}_{Q}$. We will denote $\mathcal{R}_{y_{l}} \circ \cdots \circ \mathcal{R}_{y_{2}} \circ \mathcal{R}_{y_{1}}$ by $\mathcal{R}_{\left(y_{1}, y_{2}, \ldots, y_{l}\right)}$ for $\left(y_{1}, y_{2}, \ldots, y_{l}\right) \in Q^{l}$. For quandles $Q$ and $Q^{\prime}$, a map $\phi: Q \rightarrow Q^{\prime}$ is called a quandle homomorphism if $\phi(x * y)=\phi(x) * \phi(y)$ for any $x, y \in Q$. By the condition (iii) of a quandle, for any $\mathbf{y}=\left(y_{1}, y_{2}, \ldots, y_{l}\right), \mathcal{R}_{\mathbf{y}}$ is a quandle homomorphism. 

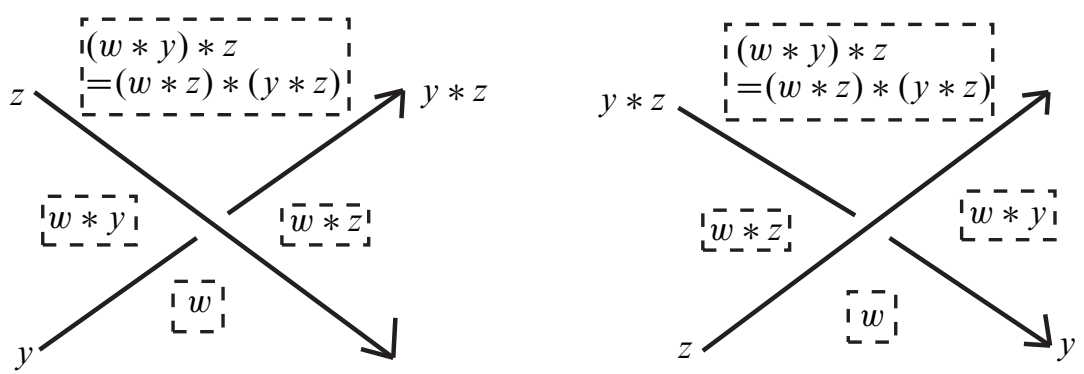

Figure 18: A shadow coloring

For a $G$-valued 3-cocycle $f$ and $\mathbf{y}=\left(y_{1}, \ldots, y_{l}\right) \in Q^{l}$, let $\widehat{f}_{\mathbf{y}}: Q^{2} \rightarrow G$ be the map defined by:

$$
\widehat{f}_{\mathbf{y}}(s, t)=\sum_{j=1}^{l} f\left(\mathcal{R}_{\left(y_{1}, \ldots, y_{j-1}\right)}(s), \mathcal{R}_{\left(y_{1}, \ldots, y_{j-1}\right)}(t), y_{j}\right)
$$

Lemma 5.1 Assume that $\mathcal{R}_{\mathbf{y}}=\mathrm{id}_{Q}$. Then the map $\widehat{f}_{\mathbf{y}}$ is a 2-cocycle.

Proof We show that $\widehat{f_{\mathbf{y}}}$ satisfies the condition of a 2-cocycle, ie

$$
\widehat{f_{\mathbf{y}}}(s, u)+\widehat{f_{\mathbf{y}}}(s * u, t * u)=\widehat{f_{\mathbf{y}}}(s, t)+\widehat{f_{\mathbf{y}}}(s * t, u),
$$

as follows. Put:

$$
F=\widehat{f_{\mathbf{y}}}(s, u)+\widehat{f_{\mathbf{y}}}(s * u, t * u)-\widehat{f_{\mathbf{y}}}(s, t)-\widehat{f_{\mathbf{y}}}(s * t, u)
$$

Since $f$ is a 3 -cocycle, $f$ satisfies:

$$
\begin{aligned}
f(s, u, v)+f(s * u, t * u, v)-f(s, t, v)-f(s * t, u, v) & \\
& =f(s * v, t * v, u * v)-f(s, t, u)
\end{aligned}
$$

As we mentioned, $\mathcal{R}_{\left(y_{1}, \ldots, y_{j-1}\right)}$ is a quandle homomorphism; thus we have:

$$
\begin{aligned}
F=\sum_{j=1}^{l}\left(f \left(\mathcal{R}_{\left(y_{1}, \ldots, y_{j-1}\right)}(s)\right.\right. & \left.* y_{j}, \mathcal{R}_{\left(y_{1}, \ldots, y_{j-1}\right)}(t) * y_{j}, \mathcal{R}_{\left(y_{1}, \ldots, y_{j-1}\right)}(u) * y_{j}\right) \\
& \left.-f\left(\mathcal{R}_{\left(y_{1}, \ldots, y_{j-1}\right)}(s), \mathcal{R}_{\left(y_{1}, \ldots, y_{j-1}\right)}(t), \mathcal{R}_{\left(y_{1}, \ldots, y_{j-1}\right)}(u)\right)\right)
\end{aligned}
$$

Since $\mathcal{R}_{\left(y_{1}, \ldots, y_{j-1}\right)}(s) * y_{j}=\mathcal{R}_{\left(y_{1}, \ldots, y_{j}\right)}(s)$, we have

$$
F=f\left(\mathcal{R}_{\mathbf{y}}(s), \mathcal{R}_{\mathbf{y}}(t), \mathcal{R}_{\mathbf{y}}(u)\right)-f\left(\mathcal{R}_{\varnothing}(s), \mathcal{R}_{\varnothing}(t), \mathcal{R}_{\varnothing}(u)\right)
$$

which is zero from $\mathcal{R}_{\varnothing}=\mathrm{id}$ and the assumption $\mathcal{R}_{\mathbf{y}}=\mathrm{id}$. Thus $\widehat{f}_{\mathbf{y}}$ is a 2 -cocycle.

For $\mathbf{x}=\left(x_{1}, \ldots, x_{m}\right)$ and $\mathbf{x}^{\prime}=\left(x_{1}^{\prime}, \ldots, x_{m}^{\prime}\right)$, let us denote $\left(x_{1}, \ldots, x_{m}, x_{1}^{\prime}, \ldots, x_{m}^{\prime}\right)$ by $\mathbf{x} \mathbf{x}^{\prime}$. 
Theorem 5.2 For a given $Q$-coloring of $\hat{b}$, let $x_{i}(i=1,2, \ldots, m)$ be the color of the $i$-th initial arc of the $m$-braid $b$. Put $\mathbf{x}=\left(x_{1}, \ldots, x_{m}\right)$, and put $\mathbf{y}=\mathbf{x}_{1} \mathbf{x}_{2} \cdots \mathbf{x}_{n}$, where $\mathbf{x}_{1}=\mathbf{x}$ and $\mathbf{x}_{j}=\mathcal{R}_{\mathbf{x}_{j-1}}\left(\mathbf{x}_{j-1}\right)(j>1)$. Assume that for any $C \in \operatorname{Col}_{Q}(\widehat{b}), \mathcal{R}_{\mathbf{y}}=\operatorname{id}_{Q}$. Then the quandle cocycle invariant of $\mathcal{S}_{m}\left(b, \Delta^{2 n}\right)$ associated with a 3 -cocycle $f$ is presented by

$$
\Phi_{f}\left(\mathcal{S}_{m}\left(b, \Delta^{2 n}\right)\right)=\sum_{C \in \operatorname{Col}_{Q}(\widehat{b})} \Phi \widehat{f}_{\mathbf{y}}(\widehat{b} ; C) \cdot \prod_{i=1}^{m} \prod_{j=1}^{n} \Psi_{f}^{*}\left(\widehat{b} ; \mathcal{R}_{\mathbf{x}}^{j-1}(C), \mathcal{R}_{\mathbf{x}}^{j-1}\left(x_{i}\right)\right)^{-1}
$$

where $\Phi \widehat{f}_{\mathbf{y}}(\widehat{b} ; C)$ is the quandle cocycle invariant of $\hat{b}$, and $\Psi_{f}^{*}(\hat{b} ; C, x)$ is the shadow cocycle invariant of $\hat{b}$. Here $\mathbf{x}$ is determined from $C$ and $b$, and $\hat{f}_{\mathbf{y}}$ is the 2-cocycle determined from $f$ and $\mathbf{y}$ by (5-1).

\subsection{Proof of Theorem 5.2}

5.2.1 Triple points of $\mathcal{S}_{m}\left(b, \Delta^{2 n}\right)$ Regarding the tubular neighborhood $N(T)$ of $T$ as $I \times I \times T$, we take for the surface diagram $D$ of $\mathcal{S}_{m}\left(b, \Delta^{2 n}\right)$ the image of the braided surface by the projection to $I \times T \subset \mathbb{R}^{3} \times\{0\}$. Cutting $N(T)$ by $p_{T}^{-1}(\mathbf{m} \cup \mathbf{l})$, we can see that $\mathcal{S}_{m}\left(b, \Delta^{2 n}\right)$ is described by a braided surface over a 2 -disk presenting $b \cdot \Delta^{2 n} \cdot b^{-1} \cdot \Delta^{-2 n} \rightarrow e$; thus $b \cdot \Delta^{2 n} \rightarrow \Delta^{2 n} \cdot b$, where we use the same notation $c$ for a diagram of a classical braid $c$. Thus the triple points of $D$ appear when we slide $b$ along $\Delta^{2 n}$, ie when we transform $b \cdot \Delta^{2 n}$ to $\Delta^{2 n} \cdot b$ fixing the diagram of $\Delta^{2 n}$. Each triple point appears when a Reidemeister move of type III occurs. Since the braid $\Delta^{2}$ is isotopic relative the boundary to the form as in Figure 19, $\mathcal{S}_{m}\left(b, \Delta^{2 n}\right)$ is equivalent to the form such that the basis braid $\Delta^{2 n}$ is $n$ powers of $\Delta^{2}$ as in Figure 19. Since equivalent surface links have the same quandle cocycle invariant, we can assume that the diagram of $\Delta^{2}$ is as in Figure 19. Sliding $b$ along $\Delta^{2 n}$ is equal to sliding $b$ along $\Delta^{2}$ $n$ times. When we slide $b$ through the $j$-th $\Delta^{2}(j=1,2, \ldots, n)$, a crossing $r$ of $b$ slides over $m$ arcs, and then under $m$ arcs of $\Delta^{2}$ (see Figure 20). Each time when $r$ slides over or under an arc, a Reidemeister move of type III occurs; thus a triple point of $D$ appears. Let $t_{1}^{j,+}(r), \ldots, t_{m}^{j,+}(r), t_{1}^{j,-}(r), \ldots, t_{m}^{j,-}(r)$ be the triple points which appear in this order.

For a given 3-cocycle $f$ and a $Q$-coloring $C$, we have the following lemma. Before a crossing $r$ slides over an arc, around $r$ there are three strings. Two strings form $r$, and they separate the other string into three arcs. According to the orientation, let us call the first arc of the three arcs the initial arc over which $r$ slides. For the colors $x$ and $y$ as in Figure 16, we call the pair $(x, y)$ the color of the crossing $r$ by $C$ (see Carter, Jelsovsky, Kamada, Langford and Saito [11]). 


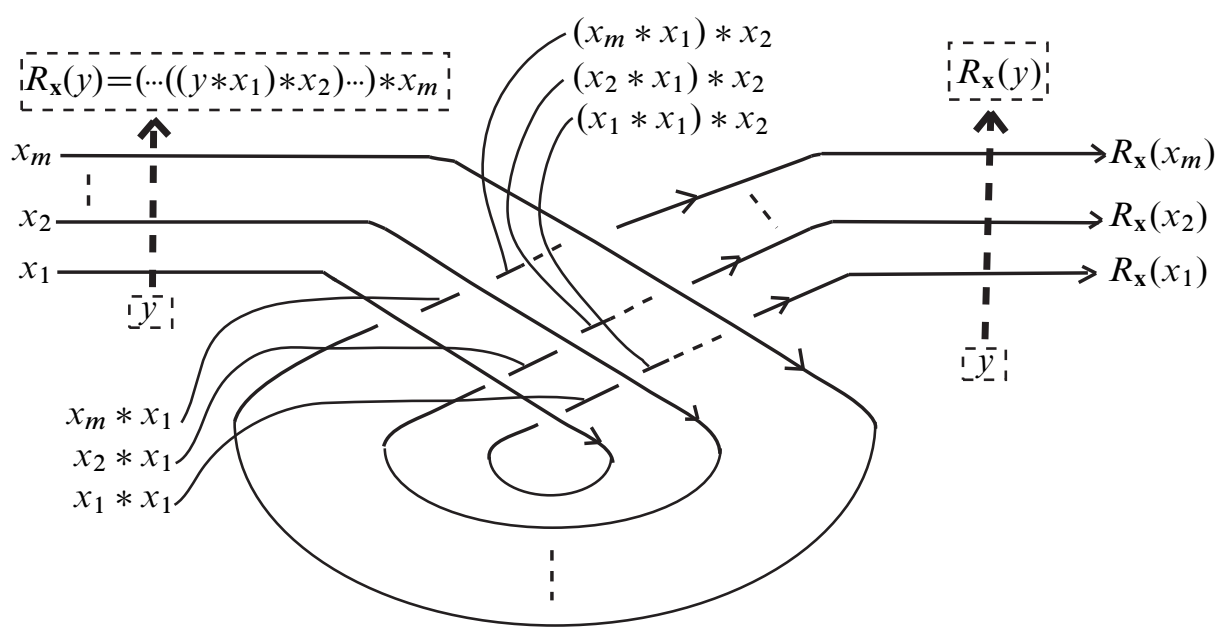

Figure 19: The braid $\Delta^{2}$

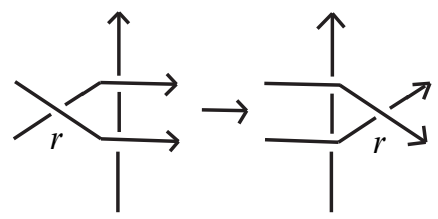

(1)

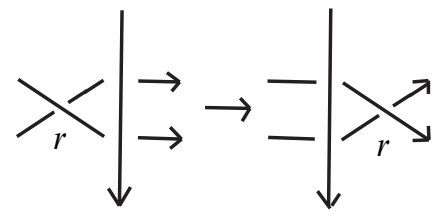

(2)

Figure 20: (1) A crossing $r$ slides over an arc, and (2) $r$ slides under an arc

Lemma 5.3 The weight of $t_{i}^{j,+}(r)$ is $f(z, x, y)^{-\epsilon}$, and the weight of $t_{i}^{j,-}(r)$ is $f(x, y, z)^{\epsilon}$, where $z$ is the color of the initial arc over or under which $r$ slides when it forms the triple point, and $(x, y)$ is the color of $r$ before sliding over or under the arc, and $\epsilon=+1$ (respectively -1 ) if $r$ is a positive (respectively negative) crossing.

Proof Put $t_{+}=t_{i}^{j,+}(r)$. If $r$ is a positive crossing, then the motion picture around $t_{+}$ is as in Figure 21. Thus, for this case, the weight of $t_{+}$is $f(z, x, y)^{-1}$; see Figure 17. If $r$ is a negative crossing, then around $t_{+}$is as in Figure 22, which is equivalent to the right figure of Figure 23. Thus the weight of $t_{+}$is $f(z, x, y)$; see Figure 17. The weight of $t_{i}^{j,-}(r)$ is obtained likewise.

5.2.2 Proof of Theorem 5.2 We take the surface diagram $D$ of $\mathcal{S}_{m}\left(b, \Delta^{2 n}\right)$ as in Section 5.2.1. First we show that $\operatorname{Col}_{Q}(D)$ and $\mathrm{Col}_{Q}(\hat{b})$ has one-to-one correspondence, as follows. Here, we have the assumption that for any $C \in \operatorname{Col}_{Q}(\widehat{b}), \mathcal{R}_{\mathbf{y}}=$ id. For a given $Q$-coloring $C$ of $D$, by restricting $C$ to the diagram of the closure of 


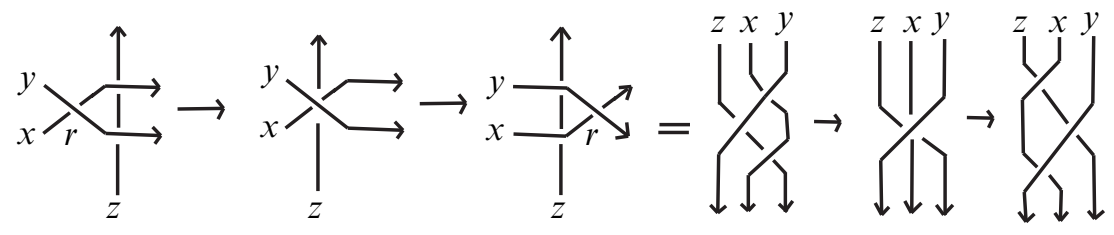

Figure 21: Around $t_{+}$if $r$ is a positive crossing

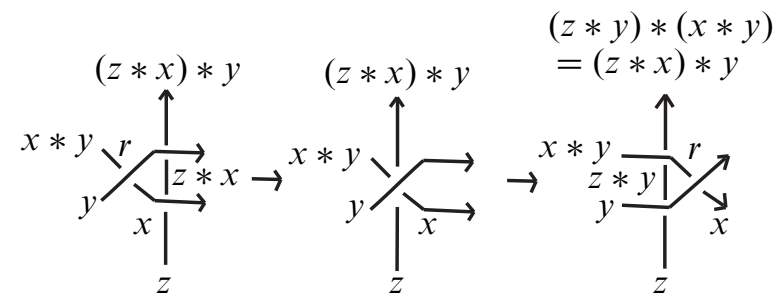

Figure 22: Around $t_{+}$if $r$ is a negative crossing
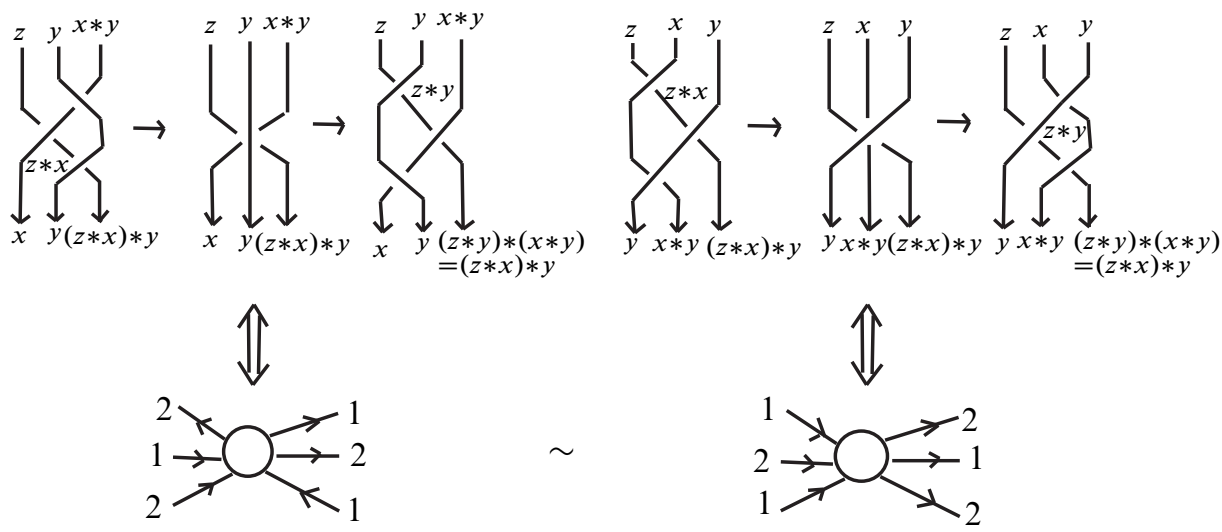

Figure 23: The motion picture of Figure 22 and its presenting white vertex (the left figure) are equivalent to those of the right figure

the basis braid $b$, we have a $Q$-coloring of $\hat{b}$. Conversely, let us consider a given $C \in \operatorname{Col}_{Q}(\hat{b})$. Let us give the other basis braid $\Delta^{2 n}$ a $Q$-coloring such that the colors of the initial arcs are $\mathbf{x}$. Since the color of the $i-$ th initial arc of the $j-$ th $\Delta^{2}$ is the $i$-th element of $\mathbf{x}_{j}$ by Lemma 5.4, it follows that $\mathcal{R}_{\mathbf{y}}(\mathbf{x})$ are the colors of the terminal $\operatorname{arcs}$ of $\Delta^{2 n}$. Since $\mathcal{R}_{\mathbf{y}}=\mathrm{id}, C$ can be extended uniquely to the diagram of the closure of $\Delta^{2 n}$; thus to the closures of the basis braids of $\mathcal{S}_{m}\left(b, \Delta^{2 n}\right)$. Since $\mathcal{S}_{m}\left(b, \Delta^{2 n}\right)$ is 
determined from the basis braids by Lemma 2.8 (2), $C$ can be extended uniquely to the surface diagram $D$.

Now we show the required formula, as follows. By definition:

$$
\Phi_{f}\left(\mathcal{S}_{m}\left(b, \Delta^{2 n}\right) ; C\right)=\prod_{r \in X_{2}(\hat{b})} \prod_{i=1}^{m} \prod_{j=1}^{n}\left(W_{f}\left(t_{i}^{j,+}(r) ; C\right) \cdot W_{f}\left(t_{i}^{j,-}(r) ; C\right)\right)
$$

First we calculate

$$
\prod_{r \in X_{2}(\widehat{b})} \prod_{i=1}^{m} \prod_{j=1}^{n} W_{f}\left(t_{i}^{j,+}(r) ; C\right),
$$

as follows. Since the color of the $i$-th initial arcs of the $j$-th $\Delta^{2}$ is $\mathcal{R}_{\mathbf{x}}^{j-1}\left(x_{i}\right)$ ( $i=1,2, \ldots, m, j=1,2, \ldots, n)$ by Lemma 5.4 , it follows that the $Q$-coloring of $b$ before sliding the $j$-th $\Delta^{2}$ is $\mathcal{R}_{\mathbf{x}}^{j-1}(C)$. The color of a crossing $r \in X_{2}(\hat{b})$ does not change when $r$ slides over an arc. When $r$ forms the triple point $t_{i}^{j,+}(r)$, the color of the initial arc over which $r$ slides is the color $w$ depicted in Figure 18, determined from a shadow coloring extending $\mathcal{R}_{\mathbf{x}}^{j-1}(C)$ with the base color which is the color of the $(m+1-i)$-th initial arc of the $j-$ th $\Delta^{2}$, ie with the base color $\mathcal{R}_{\mathbf{x}}^{j-1}\left(x_{m+1-i}\right)$ by Lemma 5.4; see Figure 24. Thus

$$
W_{f}\left(t_{i}^{j,+}(r) ; C\right)=W_{f}^{*}\left(r ; \mathcal{R}_{\mathbf{x}}^{j-1}(C), \mathcal{R}_{\mathbf{x}}^{j-1}\left(x_{m+1-i}\right)\right)^{-1}
$$

by Lemma 5.3, so:

$$
\prod_{r \in X_{2}(\hat{b})} \prod_{i=1}^{m} W_{f}\left(t_{i}^{j,+}(r) ; C\right)=\prod_{i=1}^{m} \Psi_{f}^{*}\left(\widehat{b} ; \mathcal{R}_{\mathbf{x}}^{j-1}(C), \mathcal{R}_{\mathbf{x}}^{j-1}\left(x_{i}\right)\right)^{-1}
$$

Hence:

$$
\prod_{r \in X_{2}(\hat{b})} \prod_{i=1}^{m} \prod_{j=1}^{n} W_{f}\left(t_{i}^{j,+}(r) ; C\right)=\prod_{i=1}^{m} \prod_{j=1}^{n} \Psi_{f}^{*}\left(\hat{b} ; \mathcal{R}_{\mathbf{x}}^{j-1}(C), \mathcal{R}_{\mathbf{x}}^{j-1}\left(x_{i}\right)\right)^{-1}
$$

Next we calculate

$$
\prod_{r \in X_{2}(\hat{b})} \prod_{i=1}^{m} \prod_{j=1}^{n} W_{f}\left(t_{i}^{j,-}(r) ; C\right),
$$

as follows. For each crossing $r \in X_{2}(\hat{b})$, the color $(x, y)$ of $r$ by $C$ changes to $(x * z, y * z)$, ie the color by $\mathcal{R}_{z}(C)$, when $r$ slides under an arc as in Figure 20 (2), where $z$ is the color of the arc under which $r$ slides. Let us denote the $k$-th element of $\mathbf{y}=\mathbf{x}_{1} \cdots \mathbf{x}_{n}$ by $y_{k}(k=1,2, \ldots, m n)$. When $r$ slides under the $i$-th initial arc of the 


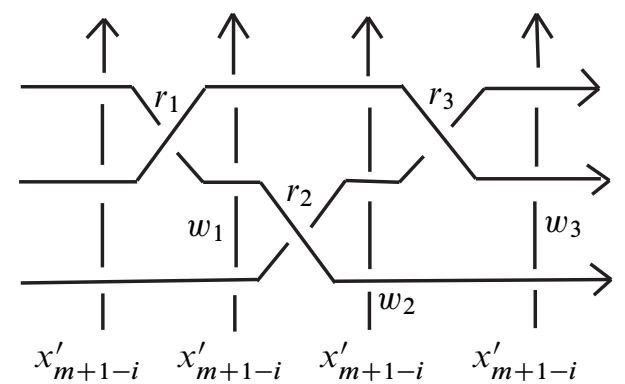

where

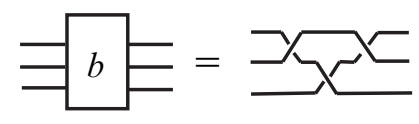

Figure 24: The color $w_{k}$ of the initial arc over which a crossing $r_{k}$ slides, when it forms $t_{i}^{j,+}\left(r_{k}\right)$, where $x_{m+1-i}^{\prime}$ is the color of the $(m+1-i)-$ th initial arc of the $j-$ th $\Delta^{2}$

$j$-th $\Delta^{2}, r$ has slid under the arcs from the first initial arc of the first $\Delta^{2}$ to the $(i-1)-$ th initial arc of the $j-$ th $\Delta^{2}$, whose colors are presented by $\left(y_{1}, y_{2}, \ldots, y_{m(j-1)+i-1}\right)$ by Lemma 5.4. Thus, when $r$ forms the triple point $t_{i}^{j,-}(r)$, the color of $r$ before sliding under an arc is the color by $\mathcal{R}_{\left(y_{1}, \ldots, y_{m(j-1)+i-1}\right)}(C)$. The arc under which $r$ slides is the $i$-th initial arc of the $j$-th $\Delta^{2}$; thus its color is the $i$-th element of $\mathbf{x}_{j}$, ie $y_{m(j-1)+i}$, by Lemma 5.4. Hence it follows from Lemma 5.3 that $W_{f}\left(t_{i}{ }^{j,-}(r) ; C\right)=$ $f\left(\mathcal{R}_{\left(y_{1}, \ldots, y_{k-1}\right)}(x), \mathcal{R}_{\left(y_{1}, \ldots, y_{k-1}\right)}(y), y_{k}\right)^{\epsilon}$, where $k=m(j-1)+i$ and $(x, y)$ is the color of $r$ by $C$, and $\epsilon=+1$ (respectively -1$)$ if $r$ is a positive (respectively negative) crossing; thus

$$
\prod_{i=1}^{m} \prod_{j=1}^{n} W_{f}\left(t_{i}^{j,-}(r) ; C\right)=W_{\widehat{y}}(r ; C),
$$

and we have:

$$
\prod_{r \in X_{2}(\widehat{b})} \prod_{i=1}^{m} \prod_{j=1}^{n} W_{f}\left(t_{i}^{j,-}(r) ; C\right)=\Phi{\widehat{f_{\mathbf{y}}}}(\widehat{b} ; C)
$$

Hence we have the required formula.

Lemma 5.4 In the situation of Theorem 5.2, the color of the $i$-th initial arc of the $j-$ th $\Delta^{2}$ is the $i$-th element of $\mathbf{x}_{j}$. Further, $\mathbf{x}_{j}=\mathcal{R}_{\mathbf{x}}^{j-1}(\mathbf{x})$.

Proof The color of the $i$-th initial arc of the $j$-th $\Delta^{2}$ is the $i$-th element of $\mathbf{x}$ (respectively $\mathcal{R}_{\mathbf{x}_{j-1}}\left(\mathbf{x}_{j-1}\right)$ ) if $j=1$ (respectively $j>1$ ), where $i=1,2, \ldots, m$ and $j=1,2, \ldots, n$ (see Figure 19); thus it is the $i$-th element of $\mathbf{x}_{j}$. Since there exists a unique shadow coloring for a given $Q$-coloring and a base color (see Carter, Kamada and Saito [13]), $\mathcal{R}_{\mathcal{R}_{\mathbf{x}}(\mathbf{x})}(y)=\mathcal{R}_{\mathbf{x}}(y)$ for any $y \in Q$; see Figure 19 . Thus $\mathbf{x}_{j}=\mathcal{R}_{\mathbf{x}}\left(\mathbf{x}_{j-1}\right)=\mathcal{R}_{\mathbf{x}}^{j-1}(\mathbf{x})$. 


\subsection{Concrete calculations}

The dihedral quandle of order $p$, denoted by $R_{p}$, is the set $\{0,1, \ldots, p-1\}$ with the binary operation $x * y=2 y-x(\bmod p)$. Mochizuki [27] showed that for any odd prime $p$, the 3 -cocycles for $R_{p}$ with the coefficient group $\mathbb{Z} / p \mathbb{Z}$ forms a group isomorphic to $\mathbb{Z} / p \mathbb{Z}$. Its generator is reduced (see Asami and Satoh [2]) to a map given by:

$$
\theta_{p}(s, t, u)=v^{(s-t)\left((2 u-t)^{p}+t^{p}-2 u^{p}\right) / p} \in\left\langle v \mid v^{p}=1\right\rangle=\mathbb{Z} / p \mathbb{Z}
$$

We call $\theta_{p}$ Mochizuki's 3-cocycle. We identify the group ring $\mathbb{Z}[\mathbb{Z} / p \mathbb{Z}]$ with the Laurent polynomial ring $\mathbb{Z}\left[v, v^{-1}\right] /\left(v^{p}-1\right)$.

Theorem 5.5 For an odd prime $p$, we have:

$$
\Phi_{\theta_{p}}\left(\mathcal{S}_{4}\left(\sigma_{1} \sigma_{2}^{p} \sigma_{3}, \Delta^{2 n}\right)\right)=p \sum_{i=0}^{p-1} v^{4 n i^{2}} \in \mathbb{Z}\left[v, v^{-1}\right] /\left(v^{p}=1\right)
$$

The triple point number of a surface link $S$ is the minimum number of triple points among all possible diagrams of $S$. By definition, the quandle cocycle invariant of a surface link with the triple point number zero has an integer value. Thus we have the following corollary.

Corollary 5.6 If $n$ is not divisible by $p$, the triple point number of $\mathcal{S}_{4}\left(\sigma_{1} \sigma_{2}^{p} \sigma_{3}, \Delta^{2 n}\right)$ is positive.

Proof of Theorem 5.5 Let us give a $R_{p}$-coloring for the diagram of the basis 4-braid $b=\sigma_{1} \sigma_{2}^{p} \sigma_{3}$. By the definition of a $R_{p}$-coloring, we have $x_{1}=x_{2}$ and $x_{3}=x_{4}$. We will denote the colors by $x$ and $y$ respectively; we have $\mathbf{x}=(x, x, y, y)$. By a direct calculation, we can see that

$$
(z * w) * w=z
$$

for any $z, w \in R_{p}$; thus $\mathcal{R}_{(w, w)}=\mathrm{id}$. Thus it follows that $\mathcal{R}_{\mathbf{x}}=\mathcal{R}_{(y, y)} \circ \mathcal{R}_{(x, x)}=\mathrm{id}$. Hence, by Theorem 5.2:

$$
\Phi_{\theta_{p}}\left(\mathcal{S}_{4}\left(\sigma_{1} \sigma_{2}^{p} \sigma_{3}, \Delta^{2 n}\right)\right)=\sum_{C \in \operatorname{Col}_{Q}(\hat{b})}\left(\Phi_{\hat{\theta}_{p \mathbf{x}}}(\hat{b} ; C)\right)^{n} \cdot\left(\Psi_{\theta_{p}}^{*}(\hat{b} ; C, x) \cdot \Psi_{\theta_{p}}^{*}(\hat{b} ; C, y)\right)^{-2 n}
$$

where

$$
\hat{\theta}_{p \mathbf{x}}(s, t)=\prod_{i=1}^{4} \theta_{p}\left(\mathcal{R}_{\left(x_{1}, \ldots, x_{i-1}\right)}(s), \mathcal{R}_{\left(x_{1}, \ldots, x_{i-1}\right)}(t), x_{i}\right)
$$


We calculate $\Phi_{\hat{\theta}_{p \mathbf{x}}}(\hat{b} ; C)$, as follows. Since $x_{1}=x_{2}=x$ and $x_{3}=x_{4}=y$, we can see that $\mathcal{R}_{\left(x_{1}, x_{2}\right)}=$ id and $\mathcal{R}_{\left(x_{1}, x_{2}, x_{3}\right)}=\mathcal{R}_{y}$. Thus:

$$
\hat{\theta}_{p \mathbf{x}}(s, t)=\theta_{p}(s, t, x) \cdot \theta_{p}(s * x, t * x, x) \cdot \theta_{p}(s, t, y) \cdot \theta_{p}(s * y, t * y, y) \in \mathbb{Z} / p \mathbb{Z}
$$

By a direct calculation (see Asami and Satoh [2]), we can see that $\theta_{p}$ satisfies

$$
\theta_{p}(s * u, t * u, u)=\theta_{p}(s, t, u)^{-1}
$$

for any $s, t, u \in R_{p}$. Thus $\hat{\theta}_{p \mathbf{x}}=1$, and hence $\Phi_{\hat{\theta}_{p \mathbf{x}}}(\hat{b} ; C)=1$ for any $C$.

We calculate $\Psi_{\theta_{p}}^{*}(\hat{b} ; C, x) \cdot \Psi_{\theta_{p}}^{*}(\hat{b} ; C, y)$, as follows. In [2], they calculated that $\Psi_{\theta_{p}}^{*}(\hat{b} ; C, x)=v^{-(x-y)^{2}}$, using the diagram of the right figure of Figure 25. Since
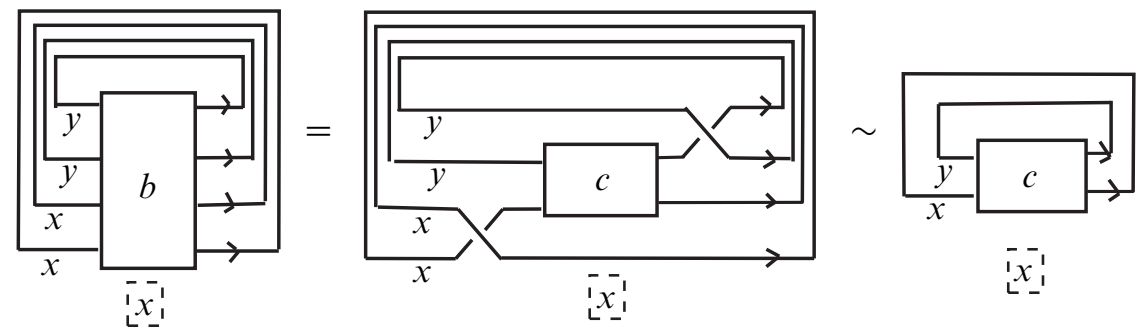

where

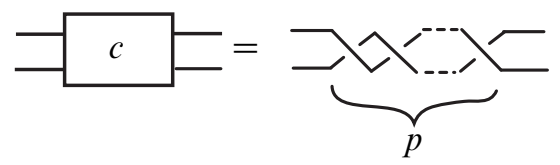

Figure 25: The shadow coloring for $\hat{b}$ with the base color $x$

the diagram of $\hat{b}$ with the $R_{p}$-coloring $C$ with the base color $y$ is transformed as in Figure 26 by Reidemeister moves, $\Psi_{\theta_{p}}^{*}(\hat{b} ; C, y)=v^{-(y-x * y)^{2}}$, which equals $v^{-(y-(2 y-x))^{2}}=v^{-(x-y)^{2}}$. Thus $\Psi_{\theta_{p}}^{*}(\hat{b} ; C, x) \cdot \Psi_{\theta_{p}}^{*}(\hat{b} ; C, y)=v^{-2(x-y)^{2}}$.

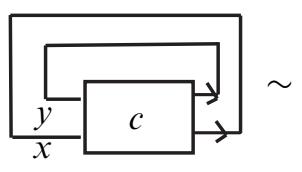

$\bar{y}$

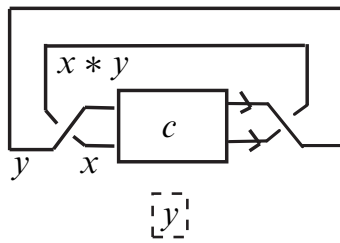

L

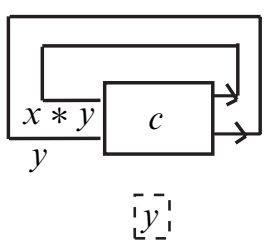

$\bar{s} \overline{y_{1}}$

Figure 26: The shadow coloring for $\hat{b}$ with the base color $y$ 
Hence, by (5-2):

$$
\begin{aligned}
\Phi_{\theta_{p}}\left(\mathcal{S}_{4}\left(\sigma_{1} \sigma_{2}^{p} \sigma_{3}, \Delta^{2 n}\right)\right) & =\sum_{x, y \in R_{p}} v^{4 n(x-y)^{2}} \\
& =p \sum_{i=0}^{p-1} v^{4 n i^{2}} \in \mathbb{Z}\left[v, v^{-1}\right] /\left(v^{p}=1\right)
\end{aligned}
$$

The quandle cocycle invariant $\Phi_{\theta_{p}}\left(\mathcal{S}_{4}\left(\sigma_{1} \sigma_{2}^{p} \sigma_{3}, \Delta^{2 n}\right)\right)$ has the same value with that of the orientation-reversed mirror image of $4 n$-twist spun $(2, p)$-torus knot $\tau^{4 n} T_{p}$ (see Asami and Satoh [2]). An oriented surface link $S$ is invertible if $S$ is equivalent to its orientation-reversed image $-S$, and (-)-amphicheiral if $S$ is equivalent to its orientation-reversed mirror image $-S^{*}$. The $4 n$-twist spun $2-k n o t \tau^{4 n} T_{p}$ is equivalent to its mirror image $\tau^{4 n} T_{p}^{*}$ (see Litherland [25]), and in [2] they showed the following fact: for an odd prime $p$ with $p \equiv 3(\bmod 4)$, if $n$ is not divisible by $p$, then $\Phi_{\theta_{p}}\left(\tau^{4 n} T_{p}\right) \neq \Phi_{\theta_{p}}\left(-\tau^{4 n} T_{p}^{*}\right)$ (see also Carter, Jelsovsky, Kamada and Saito [12]). This means that under the above conditions $\tau^{4 n} T_{p}$ is not invertible. Though $\mathcal{S}_{4}\left(\sigma_{1} \sigma_{2}^{p} \sigma_{3}, \Delta^{2 n}\right.$ ) is invertible for any $p$ and $n$ (see Proposition 2.10), comparing the quandle cocycle invariants, we have the following corollary.

Corollary 5.7 For an odd prime $p$ with $p \equiv 3(\bmod 4)$, if $n$ is not divisible by $p$, then $\mathcal{S}_{4}\left(\sigma_{1} \sigma_{2}^{p} \sigma_{3}, \Delta^{2 n}\right)$ is not (-)-amphicheiral.

Acknowledgements The author would like to thank Professors Takashi Tsuboi and Elmar Vogt for suggesting this topic, and Professors Akio Kawauchi, Tomotada Ohtsuki and the referee for their valuable advice. The author is supported by GCOE, Kyoto University.

\section{References}

[1] E Artin, Theory of braids, Ann. of Math. (2) 48 (1947) 101-126 MR0019087

[2] S Asami, S Satoh, An infinite family of non-invertible surfaces in 4-space, Bull. London Math. Soc. 37 (2005) 285-296 MR2119028

[3] I Berstein, AL Edmonds, On the construction of branched coverings of lowdimensional manifolds, Trans. Amer. Math. Soc. 247 (1979) 87-124 MR517687

[4] I Berstein, A L Edmonds, On the classification of generic branched coverings of surfaces, Illinois J. Math. 28 (1984) 64-82 MR730712

[5] J S Birman, Braids, links, and mapping class groups, Annals of Math. Studies 82, Princeton Univ. Press (1974) MR0375281 
[6] O Bogopolski, Introduction to group theory, EMS Textbooks in Math., European Math. Soc., Zürich (2008) MR2396717 Translated, revised and expanded from the 2002 Russian original

[7] J Boyle, Classifying 1-handles attached to knotted surfaces, Trans. Amer. Math. Soc. 306 (1988) 475-487 MR933302

[8] J Boyle, The turned torus knot in $S^{4}$, J. Knot Theory Ramifications 2 (1993) 239-249 MR1238874

[9] G Burde, K Murasugi, Links and Seifert fiber spaces, Duke Math. J. 37 (1970) 89-93 MR0253313

[10] G Burde, H Zieschang, Eine Kennzeichnung der Torusknoten, Math. Ann. 167 (1966) 169-176 MR0210113

[11] J S Carter, D Jelsovsky, S Kamada, L Langford, M Saito, Quandle cohomology and state-sum invariants of knotted curves and surfaces, Trans. Amer. Math. Soc. 355 (2003) 3947-3989 MR1990571

[12] J S Carter, D Jelsovsky, S Kamada, M Saito, Computations of quandle cocycle invariants of knotted curves and surfaces, Adv. Math. 157 (2001) 36-94 MR1808844

[13] J S Carter, S Kamada, M Saito, Geometric interpretations of quandle homology, J. Knot Theory Ramifications 10 (2001) 345-386 MR1825963

[14] J S Carter, M Saito, Knotted surfaces and their diagrams, Math. Surveys and Monogr. 55, Amer. Math. Soc. (1998) MR1487374

[15] E Fadell, L Neuwirth, Configuration spaces, Math. Scand. 10 (1962) 111-118 MR0141126

[16] H Gluck, The embedding of two-spheres in the four-sphere, Trans. Amer. Math. Soc. 104 (1962) 308-333 MR0146807

[17] J Hillman, 2-knots and their groups, Australian Math. Soc. Lecture Ser. 5, Cambridge Univ. Press (1989) MR1001757

[18] M W Hirsch, Differential topology, Graduate Texts in Math. 33, Springer, New York (1994) MR1336822 Corrected reprint of the 1976 original

[19] D Joyce, A classifying invariant of knots, the knot quandle, J. Pure Appl. Algebra 23 (1982) 37-65 MR638121

[20] S Kamada, Surfaces in $\mathbf{R}^{4}$ of braid index three are ribbon, J. Knot Theory Ramifications 1 (1992) 137-160 MR1164113

[21] S Kamada, 2-dimensional braids and chart descriptions, from: "Topics in knot theory (Erzurum, 1992)”, (M E Bozhuyuk, editor), NATO Adv. Sci. Inst. Ser. C Math. Phys. Sci. 399, Kluwer, Dordrecht (1993) 277-287 MR1257915

[22] S Kamada, A characterization of groups of closed orientable surfaces in 4-space, Topology 33 (1994) 113-122 MR1259518 
[23] S Kamada, An observation of surface braids via chart description, J. Knot Theory Ramifications 5 (1996) 517-529 MR1406718

[24] S Kamada, Braid and knot theory in dimension four, Math. Surveys and Monogr. 95, Amer. Math. Soc. (2002) MR1900979

[25] R A Litherland, Symmetries of twist-spun knots, from: "Knot theory and manifolds (Vancouver, B.C., 1983)”, (D Rolfsen, editor), Lecture Notes in Math. 1144, Springer, Berlin (1985) 97-107 MR823283

[26] C Livingston, Stably irreducible surfaces in $S^{4}$, Pacific J. Math. 116 (1985) 77-84 MR769824

[27] T Mochizuki, Some calculations of cohomology groups of finite Alexander quandles, J. Pure Appl. Algebra 179 (2003) 287-330 MR1960136

[28] J M Montesinos, On twins in the four-sphere. I, Quart. J. Math. Oxford Ser. (2) 34 (1983) 171-199 MR698205

[29] H Neumann, Generalized free products with amalgamated subgroups. II, Amer. J. Math. 71 (1949) 491-540 MR0030522

[30] L Rudolph, Braided surfaces and Seifert ribbons for closed braids, Comment. Math. Helv. 58 (1983) 1-37 MR699004

[31] M Teragaito, Symmetry-spun tori in the four-sphere, from: "Knots 90 (Osaka, 1990)", (A Kawauchi, editor), de Gruyter, Berlin (1992) 163-171 MR1177421

[32] T Yanagawa, On ribbon 2-knots: The 3-manifold bounded by the 2-knots, Osaka J. Math. 6 (1969) 447-464 MR0266193

Research Institute for Mathematical Sciences, Kyoto University

Oiwake-cho, Kitashirakawa, Sakyo-ku, Kyoto 606-8502, Japan

inasa@kurims.kyoto-u.ac.jp

Received: 25 June $2009 \quad$ Revised: 1 March 2011 